\title{
Regulation of Interleukin-6 Promoter Activation in Gastric Epithelial Cells Infected with Helicobacter pylori
}

\author{
Hong Lu, ${ }^{*}$ Jeng Yih Wu, ${ }^{*+}$ Takahiko Kudo, ${ }^{*}$ Tomoyuki Ohno, ${ }^{\ddagger}$ \\ David Y. Graham,* and Yoshio Yamaoka*
}

\author{
*Department of Medicine, Michael E. DeBakey Veterans Affairs Medical Center and Baylor College of \\ Medicine, Houston, TX 77030; and ‡Department of Gastroenterology and Hepatology, Kyoto Prefectural \\ University of Medicine, Kyoto 602-0841, Japan
}

Submitted May 16, 2005; Revised June 24, 2005; Accepted July 13, 2005

Monitoring Editor: J. Silvio Gutkind

\begin{abstract}
The regulation of Helicobacter pylori induced interleukin (IL)-6 in the gastric epithelium remains unclear. Primary gastric epithelial cells and MKN28 cells were cocultured with $H$. pylori and its isogenic cag pathogenicity island (PAI) mutant and/or oipA mutants. H. pylori infection-induced IL-6 mRNA expression and IL-6 protein production, which was further enhanced by the cag PAI and OipA. Luciferase reporter gene assays and electrophoretic mobility shift assays showed that full IL-6 transcription required binding sites for nuclear factor- $\kappa$ B (NF- $\kappa$ B), cAMP response element (CRE), CCAAT/ enhancer binding protein (C/EBP), and activator protein (AP)-1. The cag PAI and OipA were involved in binding to NF- $\kappa$ B, AP-1, CRE, and C/EBP sites. The cag PAI activated the extracellular signal-regulated kinase (ERK) and Jun N-terminal kinase (JNK) pathways; OipA activated the p38 pathway. Transfection of dominant negative G-protein confirmed roles for Raf, Rac1, and RhoA in IL-6 induction. Overall, the cag PAI-related IL-6 signal transduction pathway involved the Ras/Raf/MEK1/2/ERK/AP-1/CRE pathway and the JNK/AP-1/CRE pathway; the OipA-related pathway is p38/AP-1/CRE and both the cag PAI and OipA appear to be involved in the RhoA/Rac1/NF- $\kappa$ B pathway. Combination of different pathways by the cag PAI and OipA will lead to the maximum IL-6 induction.
\end{abstract}

\section{INTRODUCTION}

Interleukin (IL)-6 levels are increased in the gastric mucosa in Helicobacter pylori infection (Crabtree et al., 1991; Yamaoka et al., 1996, 1997, 2001; Ando et al., 1998; Furukawa et al., 1998) and decline after H. pylori eradication (Ando et al., 1998; Yamaoka et al., 2001). Immunohistochemistry and in situ hybridization have shown that IL-6 is abundantly expressed and secreted from both gastric epithelial cells and from inflammatory cells infiltrating the gastric mucosa in $H$. pylori infection (Furukawa et al., 1998). IL-6 levels are also higher in the antral mucosa of patients with duodenal ulcer than with simple $H$. pylori gastritis, suggesting that they may play a role in disease pathogenesis.

Although these data are consistent with the notion that IL-6 may play an important role in the gastric mucosal response to $H$. pylori infection and in the development of

This article was published online ahead of print in $M B C$ in Press (http:/ / www.molbiolcell.org/cgi/doi/10.1091/mbc.E05-05-0426) on July 19, 2005.

+ Present address: Department of Medicine, Kaohsiung Medical University, Kaohsiung, Taiwan.

Address correspondence to: Yoshio Yamaoka (yyamaoka@ bcm.tmc.edu).

Abbreviations used: AP-1, activator protein-1; C/EBP, CCAAT/ enhancer binding protein; CRE, cAMP response element; CREB, CRE-binding protein; EMSA, electrophoretic mobility shift assay; ELISA, enzyme-linked immunosorbent assay; ERK, extracellular signal-regulated kinase; IL, interleukin, JNK, Jun-N-terminal kinase; MAPK, mitogen-activated protein kinase; NF, nuclear factor; PAI, pathogenicity island. clinical $H$. pylori-related disease, the signaling pathways regulating $I L-6$ gene expression in $H$. pylori infection remains largely unstudied. There have been several reports examining IL-6 induction by $H$. pylori infection from nonepithelial cells (Gobert et al., 2004), but detailed studies investigating the role of $H$. pylori infection on IL-6 induction in gastric epithelial cells are lacking. In vitro studies of the H. pylori-related perturbations in IL-6 regulation from gastric epithelial cells have possibly been hampered by the fact that IL- 6 is not induced from commonly used gastric epithelial cell lines, such as AGS and MKN45 (Crawford et al., 2003; Hwang et al., 2003).

Finally, there are no detailed studies regarding the relationship between IL-6 induction in relation to the proinflammatory virulence factors, the cag pathogenicity island (PAI) and OipA. The cag PAI is a $40-\mathrm{kb}$ genome segment that encodes $\sim 30$ genes (Censini et al., 1996). OipA is an outer membrane protein whose transcription is regulated by a slipped-strand repair mechanism (Yamaoka et al., 2000). We chose a gastric epithelial cell line based on the pattern of response of IL-6 induction to $H$. pylori and the virulence factors the cag PAI and OipA ex vivo in normal primary gastric epithelial cells. That cell line was then used to investigate $H$. pylori-induced IL-6 production from gastric epithelial cells and the regulation of $H$. pylori-induced IL-6 transcription in gastric epithelial cells.

\section{MATERIALS AND METHODS}

\section{Epithelial Cells}

Human gastric epithelial cancer cell lines MKN1, MKN7, MKN28, MKN45, MKN74, and KATO III were obtained from Riken Cell Bank (Tsukuba, Japan). The human gastric epithelial cancer cell line AGS was obtained from American Type Culture Collection (ATCC; Manassas, VA). Human gastric epithelial cancer cell lines SNU-1, SNU-16, SNU-638, and SNU-668 cells were gifts 
from Dr. Antonia R. Sepulveda (Department of Pathology, University of Pittsburgh). In addition, the nongastric (cervical) epithelial cancer cell line, HeLa cells, were obtained from ATCC. Cell lines except for KATO III were routinely maintained in a RPMI-1640 medium (Invitrogen, Carlsbad, CA) supplemented with 10\% heat-inactivated fetal bovine serum (FBS) (Invitrogen), penicillin $\mathrm{G}(100 \mathrm{U} / \mathrm{ml})$, and streptomycin $(100 \mu \mathrm{g} / \mathrm{ml})$, whereas $20 \%$ heat-inactivated FBS were used for KATO III cells.

\section{Isolation of Primary Epithelial Cells}

Primary gastric epithelial cells were isolated enzymatically from adult human stomachs using previously established methods (Tanahashi et al., 2000). Briefly, a piece of gastric mucosa $\left(\sim 3 \mathrm{~cm}^{2}\right)$ was obtained from the normal appearing mucosa of the stomach at surgery. The patients were proven to be H. pylori-negative by a combination of serology, histology and culture. The patients gave informed consent, and the study was approved by the Human Research Committee of Kyoto Prefectural University of Medicine, Kyoto, Japan. The surface mucosal layer was removed with a razor blade, immediately minced, and then incubated in Ham's F-12 culture medium containing collagenase type I $(0.2 \mathrm{mg} / \mathrm{ml}$; Invitrogen) for $10 \mathrm{~min}$. Cells from the final incubation were washed and cultured in Ham's F-12 medium supplemented with $10 \% \mathrm{FBS}$ and streptomycin $(300 \mathrm{\mu g} / \mathrm{ml})$ at $37^{\circ} \mathrm{C}$ in a humidified $5 \% \mathrm{CO}_{2}$ atmosphere. Epithelial cells were cultured in a 24 -well collagen-coated dish at a final concentration of $10^{6} \mathrm{cells} / \mathrm{ml}$ for $24 \mathrm{~h}$ before use. Cultured cells formed subconfluent monolayers within $24 \mathrm{~h}$ of the inoculation. The cultured cells in the monolayers had periodic acid-Schiff-positive material in the cytoplasm, confirming that the population consisted of mucus producing epithelial cells with at most a minimal contamination by other cells (Tanahashi et al., 2000). Each experiment used gastric cells from a different patient with individual experiments being performed by using gastric cells from a single patient. The results were pooled from three different experiments.

\section{H. pylori}

Fifty clinical isolates were used: 20 possessed an intact cag PAI and produced OipA protein (all vacA s1 type); 20 lacked the entire cag PAI and did not produce OipA protein (all vacA s2 type); 5 possessed the entire cag PAI, but not produce OipA protein ( $3 \mathrm{vacA}$ s1 and $2 \mathrm{vac} A$ s2 type); and 5 lacked the cag PAI but produced the OipA protein (2 vacA s1 and 3 vacA s2 type). These isolates were selected from the $H$. pylori stocks at the Michael E. DeBakey Veterans Affairs Medical Center (Houston, TX). The cag PAI, OipA, and vacA status were assessed as previously described (Yamaoka et al., 2002; Kudo et al., 2004). The vacA s1 type strains, but not the vacA s2 type strains have been reported to produce large amounts of vacuolating cytotoxin (VacA; Atherton et al., 1995).

We also constructed isogenic oipA mutants, cag PAI mutants, cag PAI/oipA double mutants (representing double knockout of the cag PAI and oipA gene) vacA mutants, and ureB mutants using $H$. pylori strain TN2GF4 as a parental strain (a gift from Dr. Masafumi Nakao, Takeda Chemical Industries, Osaka, Japan). This strain was reported to induce gastric cancer in Mongolian gerbils (Watanabe et al., 1998). Isogenic oipA mutants were constructed as previously described (Yamaoka et al., 2000). For isogenic mutants for the vacA gene and the ureB genes, portions of the genes were amplified by PCR and the amplified-fragment was inserted into the EcoRV site of pBluescriptSK + (Stratagene, La Jolla, CA) with the BamHI site deleted in advance. A chloramphenicol resistance gene cassette (a gift from Diane E. Taylor, University of Alberta, Edmonton, Canada) was inserted into BsmFI site of the insert DNA for the $v a c A$ and a kanamycin resistance gene cassette (a gift from Dr. Rainer Haas, Max von Pettenkofer Institut, München, Germany) was inserted into BamHI site of insert DNA for the $u r e B$ gene. For constructing the whole cag PAIdeleted mutants, regions upstream ( $h p 0518-h p 0519 ; 545,254-547,164$ base pairs: $h p$ number and location from H. pylori strain 26695: GenBank accession number: AE000511) and downstream (hp0549-hp0550; 584,570-586,563 base pairs) of the cag PAI were amplified to delete the entire cag PAI from the $H$. pylori chromosome. These fragments, separated by a chloramphenicol resistance cassette, were cloned into the T7Blue vector (Novagen, Madison, WI). A kanamycin resistance gene cassette was also inserted into $S s p I$ site of insert DNA for the oipA gene and the resulting plasmid was used for dual inactivation for the cag PAI and the oipA by selecting on a chloramphenicol and kanamycin plate. All plasmids (1-2 $\mu \mathrm{g})$ were used for inactivation of chromosomal genes by natural transformation as previously described (Heuermann and Haas, 1998; Yamaoka et al., 2000). Inactivation of the genes was confirmed by PCR amplification followed by Southern blot as well as by Western blot for OipA (Kudo et al., 2004), CagA, VacA, and Urease (Austral Biologicals, San Ramon, CA).

H. pylori were cultured on brain heart infusion agar plates containing $7 \%$ horse blood and incubated at $37^{\circ} \mathrm{C}$ under microaerophilic conditions for 24-36 h. The cells were suspended in phosphate-buffered saline (PBS) and the density was estimated by spectrophotometry $\left(A_{625}\right)$ and by microscopic observation. We used a multiplicity of infection (MOI) of 100 to eliminate confounding effects of reduced adherence by the oipA mutants (Yamaoka et al., 2004). To avoid the influence of serum, epithelial cells were serum starved for $8 \mathrm{~h}$ (primary cells) or $16 \mathrm{~h}$ (other cells) before and throughout the period of treatment in all experiments.
In some experiments, heat-killed H. pylori (strain TN2GF4) were used at the same MOI. H. pylori were heat-killed by boiling for $15 \mathrm{~min}$ in PBS and centrifuged, and the pellets were washed three times in PBS. Killing was confirmed by lack of growth on blood agar plates. In some experiments, the same concentration of live bacteria was added to the upper well of a transwell plate (Falcon, Lincoln Park, NJ). The lower well contained subconfluent epithelial cells. In some experiments, cells were pretreated (30 min before $H$. pylori infection) with the mitogen-activated protein kinase (MAPK) inhibitor (U0126, SB203580, or SP600125) or the proteasome inhibitor, N-cbz-Leu-Leuleucinal MG-132 (MG-132; Calbiochem, San Diego, CA). U0126 is a specific inhibitor of MEK1/2 (MAPK/extracellular signal-regulated kinase [ERK] 1/2), which is located upstream of ERK1/2. SB203580 is a specific inhibitor of p38, SP600125 is a specific inhibitor of the Jun N-terminal kinase (JNK), and MG-132 inhibits nuclear factor- $\kappa$ B (NF- $\kappa$ B) activation.

\section{Small Interfering RNA}

As MG-132 is not a specific inhibitor for NF- $\kappa \mathrm{B}$, we also used Small Interfering RNA (siRNA) to interfere with NF- $\kappa$ B mRNA. MKN28 cells were transfected with TranSilent shRNA Vectors for NF- $\kappa$ B p50 and NF- $\kappa$ B p65 (0.75 $\mu \mathrm{g}$ for each) or $1.5 \mu \mathrm{g}$ of empty vectors (Panomics, Redwood City, CA) using Lipofectamine 2000 reagent (Invitrogen) according to the manufacturer's instructions. Forty hours after transfection, the medium was changed and the cells were stimulated by $H$. pylori or without $H$. pylori (negative control). In each experiment, a series of wells was dedicated to the evaluation of the silencing of the NF- $\kappa$ B by immunoblot analysis using antibodies against NF- $\kappa$ B. The level of $\beta$-actin was used as a control. In our laboratory, $\sim 80 \%$ knockdown was obtained (unpublished data).

\section{IL-6 Protein Levels from Human Epithelial Cells Cocultured with H. pylori}

In vitro IL-6 levels from human epithelial cells were examined as previously described (Yamaoka et al., 1997). Briefly, H. pylori were added to the exponentially growing epithelial cells in 24 -well plates for 12 , 24, and $36 \mathrm{~h}$. IL-6 levels in the supernatants were determined by an enzyme-linked immunosorbent assay (ELISA) (R\&D Systems, Minneapolis, MN) in duplicate. In our laboratory, the ELISA sensitivity of IL- 6 was $\sim 5 \mathrm{pg} / \mathrm{ml}$.

\section{IL-6 mRNA Levels from Human Epithelial Cells Cocultured with $\mathrm{H}$. pylori}

$H$. pylori were added to the exponentially growing epithelial cells in 24-well plates for 1, 4, 8, and $12 \mathrm{~h}$ and total RNA were isolated using RNeasy Mini Kit (Qiagen GmbH, Hilden, Germany). After DNase treatment, $1 \mu \mathrm{g}$ of total RNA was reverse-transcribed by using the Superscript first-strand synthesis system (Invitrogen) and a 1:40 dilution of the reverse transcription (RT) reaction mixture was used for quantitative PCR. Primers and probe sets used to amplify IL-6 and the housekeeping gene ( $\beta$-actin) were purchased from Perkin Elmer-Cetus Applied Biosystems (Foster City, CA). Reaction mixtures for PCR $(50 \mu \mathrm{L}$ ) were prepared by mixing $5 \mu \mathrm{L}$ of synthesized cDNA solution with 2X TaqMan Universal PCR Master Mix (Perkin Elmer-Cetus Applied Biosystems), $500 \mathrm{nM}$ of each primer, and $250 \mathrm{nM}$ of the TaqMan probe. Real-time PCR was performed using an ABI Prism 7300 Sequence-Detection System (Perkin Elmer-Cetus Applied Biosystems) at $50^{\circ} \mathrm{C}$ for $2 \mathrm{~min}, 95^{\circ} \mathrm{C}$ for $10 \mathrm{~min}$, followed by 40 cycles of $95^{\circ} \mathrm{C}$ for $15 \mathrm{~s}$, and $60^{\circ} \mathrm{C}$ for $60 \mathrm{~s}$. Data were collected in triplicate, and IL-6 mRNA levels were expressed as the ratio of IL-6 mRNA to $\beta$-actin mRNA $(10,000 \times$ IL- 6 mRNA/ $\beta$-actin mRNA).

\section{Luciferase Plasmids}

The transcriptional regulation of the human IL-6 gene involves at least four different transcription factors: i.e., NF- $\kappa \mathrm{B}(\sim-73$ to $-64 \mathrm{nt})$, activator protein (AP)-1 ( - 283 to -277 ), CCAAT/enhancer binding protein (C/EBP; $\sim-158$ to -145 ), and cAMP response element (CRE)-binding protein (CREB; $\sim-163$ to -158; Akira, 1997). The full-size human IL-6 promoter reporter gene construct p1168hu.IL6P-luct and the point-mutated variants p1168(NF$\kappa$ Bmut).IL6P-luc+, p1168(AP-1mut).IL6P-luc+, p1168(CREmut).IL6P-luc+, p1168(C/EBP-CREmut).IL6P-luc+, and p1168(C/EBPmut).IL6P-luc+ were constructed previously (mut = mutated; Plaisance et al., 1997; Vanden Berghe et al., 1998; Vanden Berghe et al., 1999) and were purchased from BBCM/ LMBP collection (Ghent University, Belgium). The recombinant plasmids $\mathrm{p}$ (IL6 $\kappa \mathrm{B})_{3} 50$ hu.IL6P-luc + , containing multimerized IL6 NF- $\kappa$ B elements in front of p50hu.IL6-luc + (Plaisance et al., 1997), were also purchased from the BBCM/LMBP collection. The above IL-6 promoter fragments were inserted the multicloning site of pGL basic (Promega, Madison, WI; Plaisance et al., 1997; Vanden Berghe et al., 1998; Vanden Berghe et al., 1999). The 5' deletion constructs of the human IL-6 promoter were gifts from Dr. Toshio Hirano (Osaka University Medical School, Osaka, Japan), including p1160-luc+ (full size), 181-luct, p108-luct, and p36-luc+ (Muraoka et al., 1993). The IL-6 promoter fragments were inserted the multicloning site of PGV-B (Toyo, Tokyo, Japan; Muraoka et al., 1993).

The PathDetect cis-reporting plasmids pNF- $\kappa$ Bluci, which contain the luciferase reporter gene driven by the basic promoter element TATA box plus five repeats of the consensus binding sequence for the NF- $\kappa B$, were purchased 
from Stratagene (La Jolla, CA). The plasmid pCMV-RafS621A, which expresses a dominant negative form of the Raf protein when transfected into mammalian cells, was purchased from Clontech (Palo Alto, CA). The pCMVRhoN19, pCMV-RacN17, and pCMV-RasN17 expression plasmids, which express the dominant negative form of RhoA, Rac1, and Ras proteins when transfected into mammalian cells, were previously described (Coso et al. 1995; Sun et al., 2002)

\section{Cell Transfection}

Exponentially growing MKN28 cells in 24-well plates were transfected with a luciferase reporter gene plasmid of interest using Lipofectamine 2000 reagent (Invitrogen) for the luciferase reporter gene assay. In some experiments, MKN28 cells were cotransfected with the p1168hu.IL6P-luc+ plasmid plus one of the following plasmids: pCMV-RasN17, pCMV-RacN17, pCMVRhoN19, pCMV-RafS621A, or the empty vector pCMV. The luciferase reporter gene assays were performed with Dual-Luciferase reporter assay system according to the manufacturer's instructions (Promega). In this system the phRL-TK plasmid, a Renilla reniformis luciferase vector DNA (10 ng), was cotransfected for the internal controls and normalization of transfection efficiency. Thirty hours after transfection, the medium was changed and the cells were stimulated with $H$. pylori or without $H$. pylori (negative control). The cells were lysed at $3,6,9,12$, and $18 \mathrm{~h}$ and the lysates were assayed for luciferase activity. Luciferase activity was normalized to Renilla luciferase vector DNA (normalized luciferase activity). We also assessed the luciferase activity as the fold increase of luciferase activity in $\mathrm{H}$. pylori-infected cells relative to uninfected controls (fold increase). We confirmed that expression of Renilla was not induced by H. pylori infection (unpublished data).

\section{Electrophoretic Mobility Shift Assay (EMSA)}

Nuclear extracts of uninfected and infected MKN28 cells were prepared using hypotonic/nonionic detergent lysis as previously described (Brasier et al., 1998). After extraction, nuclear proteins were normalized based on the protein assay (Protein Reagent; Bio-Rad, Hercules, CA), and equal amounts were used to bind to duplex oligonucleotides corresponding to the IL-6-specific AP-1, CRE, C/EBP, and NF- $\kappa$ B binding sites. The gel shift IL-6 promoter specific oligonucleotides used in this study were $5^{\prime}$-CCA AGT GCT GAG TCA CTA ATA AAG-3' for AP-1, 5'-TGC GAT GCT AAA GGA CGT CAG GGA ACA GGC TT-3' for CRE, 5'-AAG GGG TCA TTG CAC AAT CTT AAT AAG G-3' for C/EBP, and 5'-CAA ATG TGG GAT TTT CCC ATG AGT C-3' for NF- $\kappa$ B. The mutated oligonucleotides (altered nucleotides are underlined) were 5'-CCA AGT GCT GCA GCA CTA ATA AAG-3' for AP-1, 5'-TGC GAT GCT AAA GGG ATC CAG GGA ACA GGC TT-3' for CRE, 5'-AAG GGG TCA GAT ATC AAT CTT AAT AAG G-3' for C/EBP, and 5'-CAA ATG TGA GAT CTT CCC ATG AGT C-3' for NF- $\kappa$ B. DNA-binding reactions for the $\mathrm{NF}-\kappa \mathrm{B}$ and AP-1 probes were as described previously (Yamaoka et al., 2004). Binding reactions for the CRE and C/EBP probes contained 10-15 $\mu \mathrm{g}$ total protein, $5 \%$ glycerol, $12 \mathrm{mM}$ HEPES, $80 \mathrm{mM} \mathrm{NaCl}, 1 \mathrm{mM}$ dithiothreitol, $5 \mathrm{mM}$ $\mathrm{Mg}_{2} \mathrm{Cl}, 0.5 \mathrm{mM}$ EDTA, $1 \mu \mathrm{g}$ of poly (dI-dC), and $40,000 \mathrm{cpm}$ of ${ }^{32} \mathrm{P}$-labeled double-stranded oligonucleotide in a total volume of $20 \mu \mathrm{L}$. The nuclear proteins were incubated with the probe for $15 \mathrm{~min}$ at room temperature and then fractionated on $6 \%$ nondenaturing PAGE in Tris-borate-EDTA buffer (22 $\mathrm{mM}$ Tris- $\mathrm{HCl}, 22 \mathrm{mM}$ boric acid, $0.25 \mathrm{mM}$ EDTA, $\mathrm{pH}$ 8). After electrophoretic separation, gels were dried and exposed for autoradiography using Kodak XAR film (Eastman Kodak, Rochester, NY) at $-80^{\circ} \mathrm{C}$ and intensifying screens.

For competition assays, extracts were incubated with a 100-fold excess of unlabeled competitors. Gel mobility supershift assays were performed to examine which molecules bind to the specific sites in the IL-6 promoter. We used commercial antibodies (anti-p50 [sc-1191], anti-p65 [sc-109], anti-c-Fos [sc-413], anti-c-Jun [sc-44], anti-CREB1 [sc-186], anti-CREB2 [sc-200], antiATF-2 [sc-242], anti-C/EBP- $\alpha$ [sc-61], anti-C/EBP- $\beta[$ sc-150], and anti-C/EBP$\delta$ [sc-151]; Santa Cruz Biotechnology, Santa Cruz, CA), which were added to the extracts $45 \mathrm{~min}$ before addition of the probe.

\section{Western Blot Analysis for MAPK}

Western blot analysis was performed for p38, ERK, and JNK to examine whether $H$. pylori activated their phosphorylation. We used phospho-specific antibodies to detect phospho-p38 (9211), phospho-ERK1/2 (9101) and phospho-JNK (9251) and control antibodies to unphosphorylated forms of p38 (9212), ERK1/2 (9102) and JNK (9252; Cell Signaling Technology, Beverly, MA). Exponentially growing MKN28 cells $\left(\sim 2 \times 10^{5} / \mathrm{ml}\right)$ in $10-\mathrm{cm}^{2}$ plates were either uninfected or infected with $H$. pylori for $0-3 \mathrm{~h}$. An equal amount of total protein extract was fractionated by SDS-PAGE and electrophoretically transferred to a polyvinylidene difluoride membrane. An enhanced chemiluminescence detection assay (Amersham Pharmacia Biotech, Piscataway, NY) was performed according to the Manufacturer's instructions.

\section{Statistical Analysis}

Statistical analysis was performed by the Mann-Whitney Rank Sum test and the paired $t$ test depending on the data set of concern. The data are presented as mean \pm SE. $p<0.05$ was accepted as statistically significant.
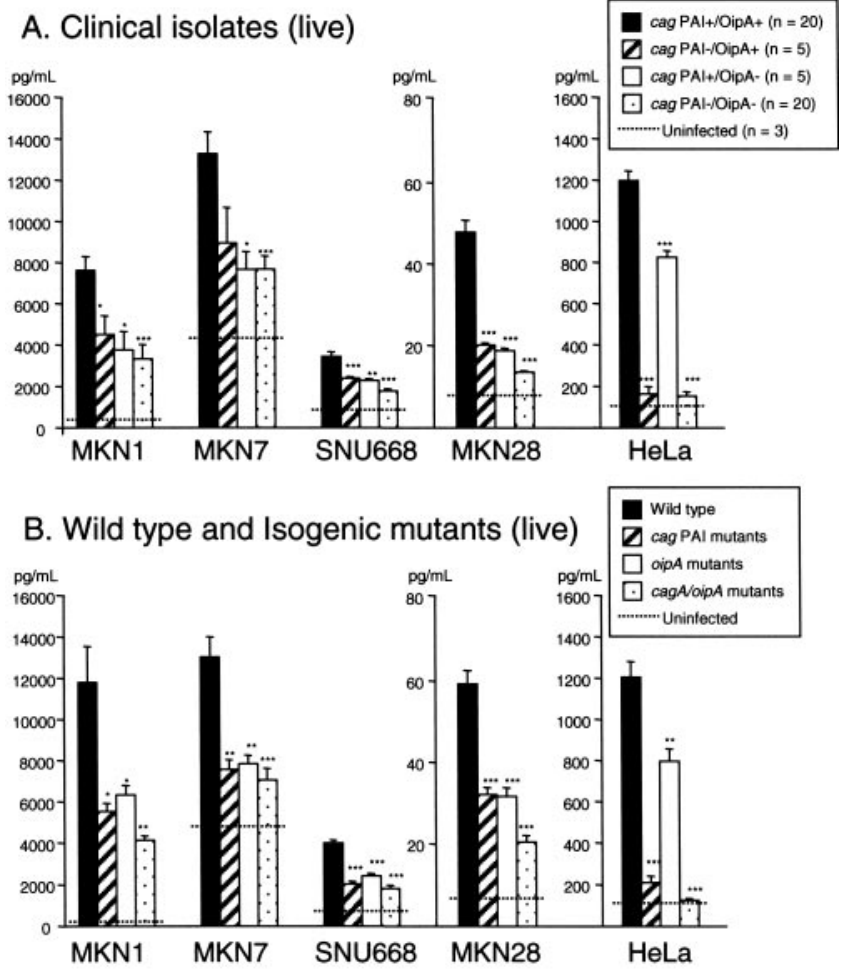

C. Wild type and Isogenic mutants (heat-killed)

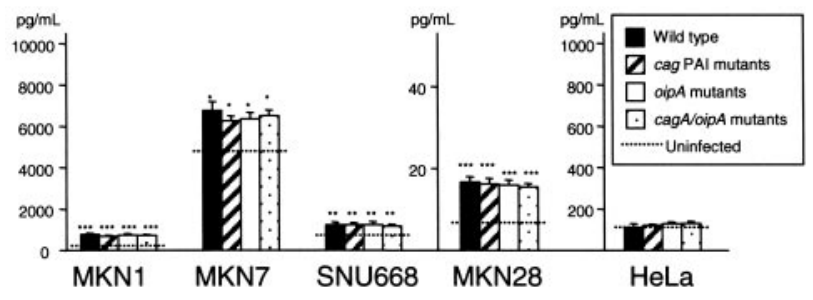

Figure 1. IL-6 protein levels from various epithelial cells infected with $H$. pylori. IL-6 levels from epithelial cells cocultured with $H$. pylori for $24 \mathrm{~h}$ were measured by ELISA in duplicate. (A) clinical isolates (live), (B) wild-type strain TN2GF4 and its isogenic mutants (live), (C) wild-type strain TN2GF4 and its isogenic mutants (heatkilled). Three (A) or four (B and C) independent coculture experiment, each done in triplicate, were performed. Data are expressed as mean $\pm \mathrm{SE}$; ${ }^{*} \mathrm{p}<0.05,{ }^{* *} \mathrm{p}<0.01$, and ${ }^{* * *} \mathrm{p}<0.001$ compared with the cag PAI-positive/OipA-positive bacteria (A) or wild-type $H$. pylori (B and $\mathrm{C})$.

\section{RESULTS}

\section{H. pylori Strains Induced IL-6 Production from Gastric Epithelial Cells and the Induction Was Both the cag PAI and OipA Dependent}

IL-6 levels were examined using 12 cell lines cocultured with 50 clinical isolates. IL-6 levels were below the level of detection with MKN45, MKN74, KATOIII, AGS, SNU-1, SNU-16, and SNU-638 cells irrespective of whether $H$. pylori was present or not (unpublished data). H. pylori induced IL-6 from MKN1, MKN7, MKN28, SNU-668, and HeLa cells with peak levels at $24 \mathrm{~h}$ (IL-6 levels at $24 \mathrm{~h}$ are shown in Figure 1A). Of interest, even among gastric cancer cells we found differences in IL-6 levels. In IL-6 producing cell lines, IL-6 induction was cag PAI and OipA dependent. However, cag PAI-negative/OipA-negative bacteria induced small but 
significantly higher amounts of IL-6 compared with the uninfected control gastric epithelial cells $(\mathrm{pg} / \mathrm{ml}=3461 \pm$ 690 vs. $250 \pm 45$ for MKN1, $7671 \pm 645$ vs. $4320 \pm 311$ for MKN7, $1768 \pm 95$ vs. $871 \pm 8$ for SNU668, and $13.9 \pm 0.3$ vs. $7.7 \pm 0.9$ for MKN28, $\mathrm{p}<0.001$ for each). In contrast, cag PAI-negative/OipA-negative bacteria did not induce IL-6 from the nongastric, HeLa cells $(154 \pm 20$ vs. $112 \pm 22$ for uninfected control; $\mathrm{p}=0.34)$. These observations were confirmed using a parental $H$. pylori strain and its isogenic mutants (Figure 1B). The reduction of IL-6 production with the cag PAI mutants was similar to that observed with the oipA mutants in gastric epithelial cells. In contrast, the influence of the cag PAI in HeLa cells was much greater than that of OipA, suggesting that the pathways to induce IL- 6 by $H$. pylori differ among tissues.

We also examined whether there was a requirement for live H. pylori for IL-6 production. As shown in Figure 1C, heat-killed bacteria were able to induce small, but significantly higher, amounts of IL-6 from gastric epithelial cells compared with the uninfected control. However, they did not stimulate IL-6 production in HeLa cells, suggesting that the heat resistant factors may only contribute to IL-6 production from gastric epithelial cells. Live $H$. pylori separated by a permeable membrane were unable to induce IL-6 (unpublished data), suggesting that soluble factors produced from $H$. pylori are not involved. We confirmed that the soluble factors, VacA and urease were specifically not involved in IL-6 induction by showing that IL-6 levels for the vac $A$ mutants and $u r e B$ mutants were similar to wild-type strains irrespective of the epithelial cell type (unpublished data).

We next examined whether primary noncancer gastric epithelial cells responded similarly to stable cells lines infected with $H$. pylori. Importantly, wild-type $H$. pylori were able to induce IL-6 from noncancer primary gastric cells (Figure 2). The reduction of IL-6 levels associated with the use of the cag PAI mutants was similar to that obtained with the oipA mutants. Similar to observations with gastric cancer cells, both the cag PAI/oipA double mutants and heat-killed $H$. pylori induced small amounts of IL-6. Thus, the pattern of IL-6 induction with the primary gastric epithelial cells was the same as that observed using gastric cancer cells such as MKN28 cells. We tested primary cells from three different individuals, and the patterns of IL-6 induction were similar among all. Because MKN28 cells provided results similar to those obtained from primary cells, MKN28 cells were used in subsequent experiments. Another advantage of MKN28 cells is the fact that plasmids were able to easily be transfected into MKN28 cell. In contrast, transfection of plasmids proved difficult with primary cells, MKN1, MKN7, and SNU668 cells (unpublished data).

\section{H. pylori-induced IL-6 Production Occurred through MAPK Pathways and NF-кB Pathways}

We evaluated the effect of inhibitors of the different signal transduction pathways for IL-6 induction. We added each inhibitor (SB203580: p38 inhibitor, U0126: MEK1/2 inhibitor, SP600125: JNK inhibitor and MG-132: NF- $\kappa$ B inhibitor; 0.5, 1, $5,10,30$, and $100 \mu \mathrm{M})$ to MKN28 cells $30 \mathrm{~min}$ before infection with the wild-type strain (Figure 3). SB203580, U0126, and JNK inhibitors did not produce any effect on IL-6 levels in uninfected MKN28 cells. In contrast, MG-132 alone had an ability to induce IL- 6 at 5 and $10 \mu \mathrm{M}$, which is consistent with previous studies showing MG-132 induced IL-6 from C2C12 myoblasts (Frost et al., 2003), umbilical vein endothelial cells (Shibata et al., 2002), and colon epithelial cells (Caco-2; Pritts et al., 2002). Of interest, MG-132, even at 1

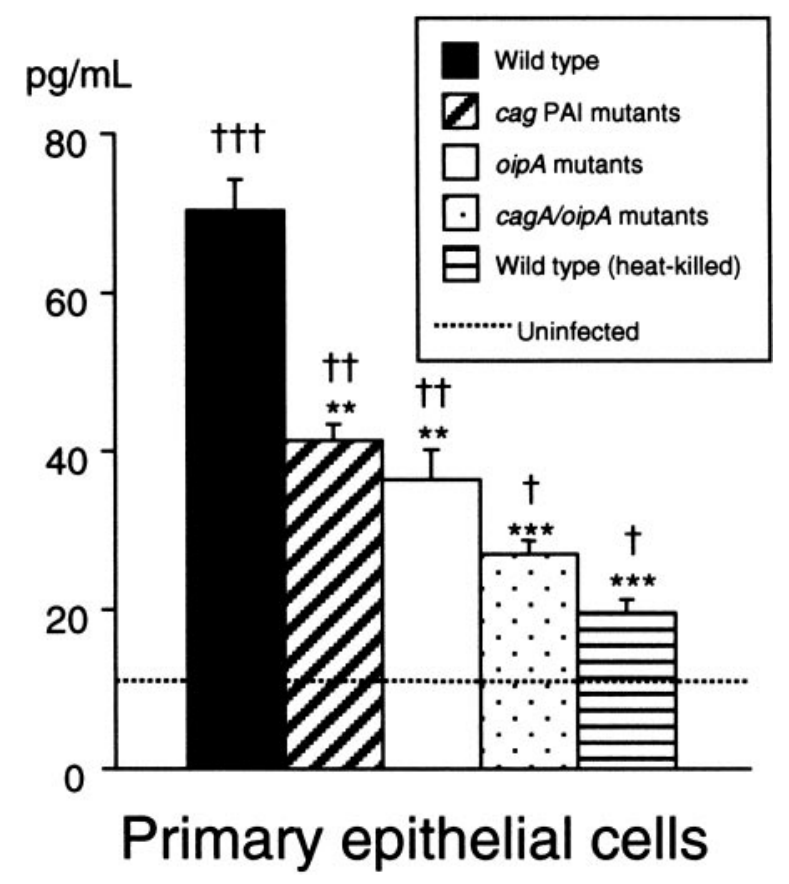

Figure 2. IL-6 protein levels from primary gastric epithelial cells infected with $H$. pylori. IL-6 levels from primary noncancer gastric epithelial cells cocultured with $H$. pylori for $24 \mathrm{~h}$ were measured by ELISA in duplicate. Three independent coculture experiments, each measured in triplicate, were performed. Data are expressed as mean \pm SE; ${ }^{* *} \mathrm{p}<0.01$ and ${ }^{* * *} \mathrm{p}<0.001$ compared with wild-type H. pylori. ${ }^{+} \mathrm{p}<0.05,{ }^{++} \mathrm{p}<0.01,{ }^{+++} \mathrm{p}<0.001$ compared with uninfected control.

$\mu \mathrm{M}$, suppressed the induction of $H$. pylori-induced IL- 6 protein markedly ( $\sim 95 \%$ reduction; Figure 3$)$. We used $1 \mu \mathrm{M}$ of MG-132 in subsequent experiments to prevent MG-132-related IL-6 induction. SB203580, U0126, and SP600125 suppressed the induction of $H$. pylori-induced IL-6 protein markedly at $10 \mu \mathrm{M}(\sim 50 \%$ reduction; Figure 3$)$. We therefore used $10 \mu \mathrm{M}$ of SB203580, U0126, and SP600125 in subsequent experiments.

We also tested the effect of the inhibitors on $H$. pyloriinduced IL-6 production using the isogenic mutants (Figure 4A). Interestingly, U0126 and SP600125 significantly suppressed IL- 6 production induced by the oipA mutants, whereas the reduction was only slight for the cag PAI mutants, suggesting that cag PAI is involved in activation of the MEK1/2 $\rightarrow$ ERK and the JNK pathways. In contrast, SB203580 significantly suppressed IL-6 production induced by the cag PAI mutants but the reduction was slight for the oipA mutants, indicating that OipA is involved in activation of the p38 pathway. It is unclear whether the small reductions of IL-6 induction associated with U0126 and SP600125 using the cag PAI mutants or the reduction of IL- 6 induction associated with SB203580 using oipA mutants has biological meaning. Interestingly, U0126 suppressed IL-6 production induced by the cag PAI/oipA double mutants or heat-killed $H$. pylori, indicating that possibly heat-resistant factors other than the cag PAI and OipA may also be involved in activation of the MEK1/2 $\rightarrow$ ERK pathway.

MG-132 significantly suppressed IL-6 production induced by the cag PAI mutants and the oipA mutants; however MG-132 did not suppress IL-6 production induced by the cag PAI/oipA double mutants. Because MG-132 is not a specific inhibitor for NF- $\kappa$ B pathway, we also used siRNA to confirm 

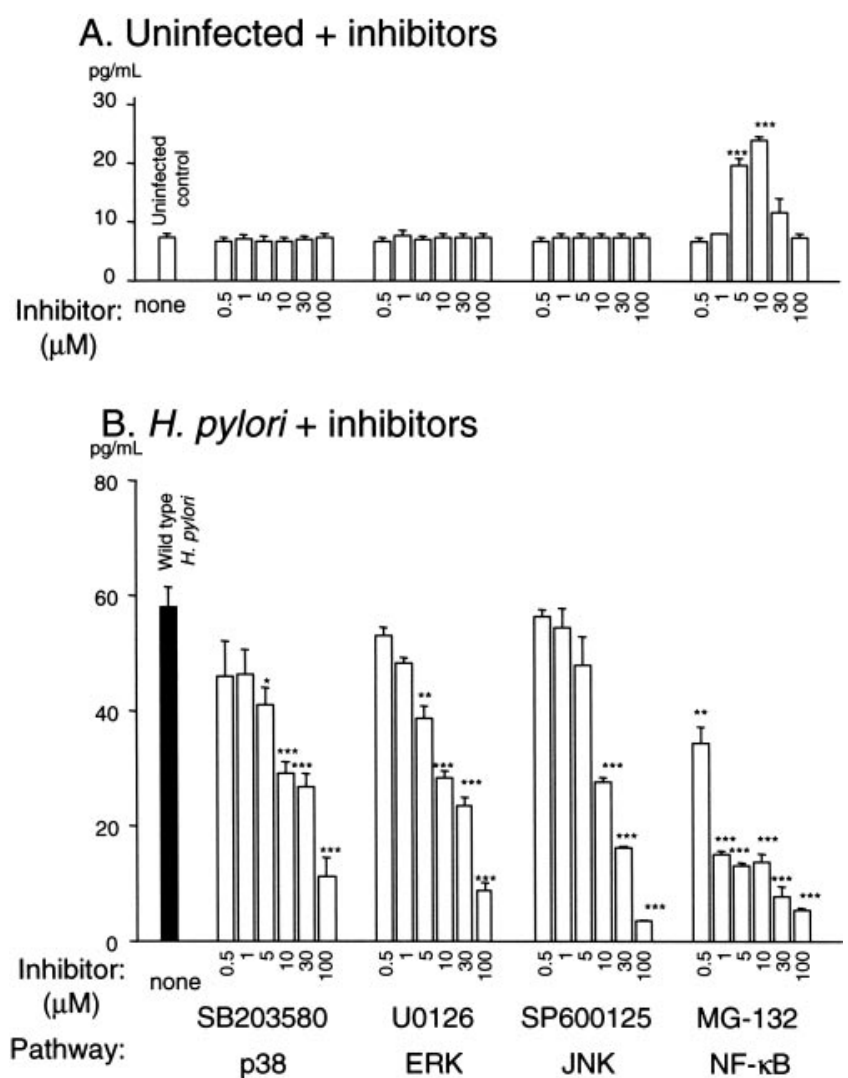

Figure 3. Effects of inhibition of MAPK pathways and NF- $\kappa$ B pathways on $H$. pylori-induced IL-6 levels in MKN28 cells. Each inhibitor $(0.5-100 \mu \mathrm{M})$ was added to MKN28 cells $30 \mathrm{~min}$ before wild-type $H$. pylori infection. Cells were then incubated for $24 \mathrm{~h}$ and IL-6 protein levels were determined by ELISA. IL-6 levels without (A) or with (B) $H$. pylori infection are presented. Four independent coculture experiments, each measured in triplicate, were performed. Data are expressed as mean $\pm S E ;{ }^{*} p<0.05,{ }^{* *} p<0.01$, and ${ }^{* * *} p<$ 0.001 compared with uninfected control (A) and wild-type H. pylori (B) without inhibitors. Data are expressed as mean \pm SE.

our data. As shown in Figure $4 \mathrm{~B}, \mathrm{NF}-\kappa \mathrm{B}$ siRNA suppressed IL-6 production induced by the cag PAI mutants and the oipA mutants as well as by wild-type $H$. pylori; however NF- $\kappa \mathrm{B}$ siRNA did not suppress IL-6 production induced by the cag PAI/oipA double mutants. These data suggest that the cag PAI and OipA are main players in activation of the $\mathrm{NF}-\kappa \mathrm{B}$ pathway.

\section{H. pylori Strains Induced IL-6 mRNA through the cag $P A I \rightarrow M E K \rightarrow E R K$ Pathway, OipA $\rightarrow$ p38 Pathway, and the cag PAI/OipA $\rightarrow$ NF-кB Pathway}

IL-6 mRNA was detectable at $1 \mathrm{~h}$ postinfection and reached maximal levels at $4 \mathrm{~h}$; data at $4 \mathrm{~h}$ are shown in Figure 5. Induction patterns of mRNA levels paralleled the protein levels confirming that $H$. pylori-induced IL-6 mRNA expression was both the cag PAI and OipA dependent and that the cag PAI/oipA double mutants and heatkilled $H$. pylori were able to induce small amounts of IL-6 mRNA. Similar to the results with protein levels, live $H$. pylori separated by a permeable membrane were unable to induce IL- 6 mRNA (unpublished data). IL- 6 mRNA levels for the vacA mutants and the ureB mutants were also

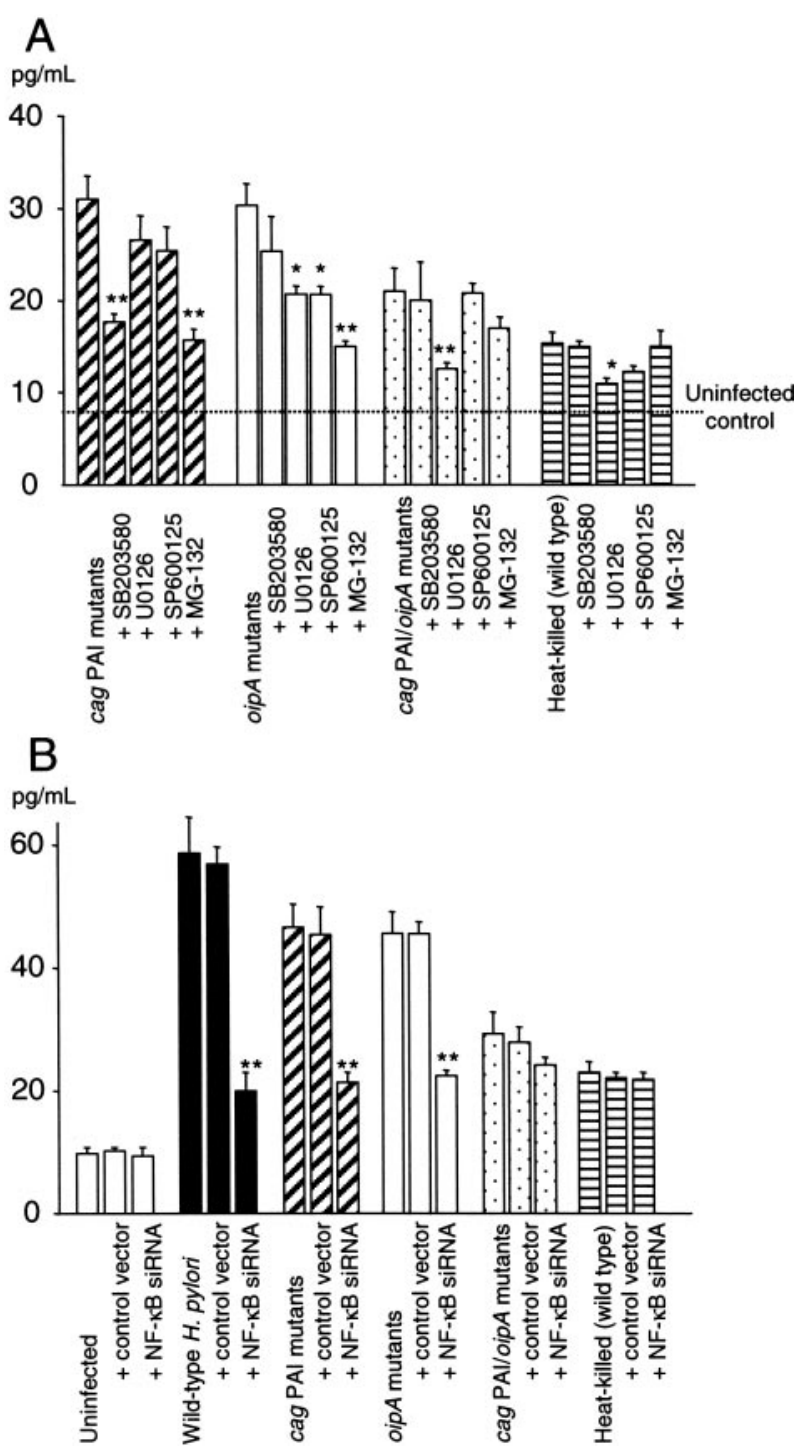

Figure 4. Effects of inhibition of MAPK pathways and NF- $\kappa$ B pathways on IL-6 levels in MKN28 cells using isogenic mutants. (A) Each inhibitor $(10 \mu \mathrm{M}$ for MAPK inhibitors and $1 \mathrm{M}$ for NF- $\kappa$ B inhibitor) was added to MKN28 cells 30 min before wildtype $H$. pylori infection. (B) MKN28 cells were transfected with TranSilent shRNA Vectors for NF- $\kappa$ B p50 and NF- $\kappa$ B p65 $(0.75 \mu \mathrm{g}$ for each) or $1.5 \mu \mathrm{g}$ of empty vectors (Panomics) and $40 \mathrm{~h}$ after transfection, the medium was changed and the cells were stimulated by $H$. pylori. In both experiments, the cells were incubated for $24 \mathrm{~h}$ after $H$. pylori infection and IL-6 protein levels were determined by ELISA. Four independent coculture experiments, each measured in triplicate, were performed. Data are expressed as mean $\pm \mathrm{SE} ;{ }^{*} \mathrm{p}<0.05$ and ${ }^{* *} \mathrm{p}<0.01$ compared with each H. pylori without inhibitors.

similar to those for wild-type strains (unpublished data). Inhibitor experiments (Figure 5) and the use of NF- $\kappa \mathrm{B}$ siRNA (unpublished data) also confirmed that the patterns of induction of mRNA levels were parallel to those of protein levels. Overall, these findings from mRNA and protein levels of IL-6 suggest that the cag PAI $\rightarrow$ MEK1/ $2 \rightarrow$ ERK pathway, OipA $\rightarrow$ p38 pathway, and the cag PAI/ $\mathrm{OipA} \rightarrow \mathrm{NF}-\kappa \mathrm{B}$ pathway are involved in IL-6 induction and that IL-6 induction by heat-killed bacteria involves the MEK1/2 $\rightarrow$ ERK pathway. 


\section{$10,000 \times$ IL-6 mRNA/ $\beta$-actin mRNA}

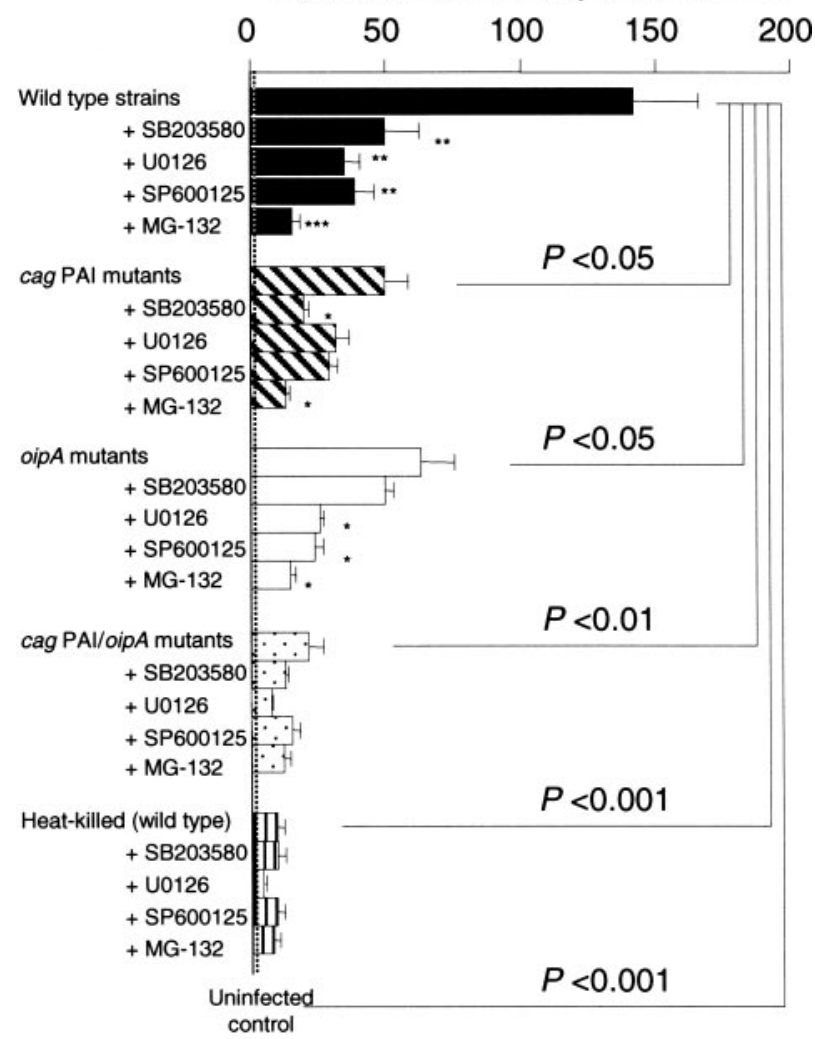

Figure 5. IL-6 mRNA levels in MKN28 cells infected with $H$. pylori and effects of inhibition of MAPK pathways and NF- $\kappa$ B pathways. Each inhibitor $(10 \mu \mathrm{M}$ for MAPK inhibitors and $1 \mathrm{M}$ for NF- $\kappa \mathrm{B}$ inhibitor) was added to MKN28 cells $30 \mathrm{~min}$ before wild-type $H$. pylori infection, then cells were incubated for $4 \mathrm{~h}$, and IL- 6 mRNA levels were determined by real-time RT-PCR. Three or four independent coculture experiments, each measured in triplicate, were performed. Data are expressed as the ratio of IL-6 mRNA to $\beta$-actin mRNA $(10,000 \times$ MMP-1 mRNAs $/ \beta$-actin mRNA). Data are expressed as mean $\pm \mathrm{SE} ;{ }^{*} \mathrm{p}<0.05,{ }^{* *} \mathrm{p}<0.01$, and ${ }^{* * *} \mathrm{p}<0.001$ compared with each $H$. pylori without inhibitors.
H. pylori Strains Induced IL-6 Promoter Activity and the Activity Was Both the cag PAI and OipA Dependent

To verify that the increase in IL-6 mRNA and protein levels was due to transcriptional regulation at the promoter level, $0.5 \mu \mathrm{g}$ of the full-length IL-6 promoter reporter plasmid p1168hu.IL6P-luc+ (from BBCM/LMBP collection) or p1160-luc + (from Dr. Hirano) were transfected in MKN28 cells. Luciferase activity reached maximal levels at $9 \mathrm{~h}$ postinfection irrespective of the H. pylori strain used (unpublished data). When we used p1168hu.IL6P-luc+, peak activity was significantly higher for the wild-type H. pylori (normalized activity by Renilla luciferase vector $=19.7 \pm 3.0$, fold increase of uninfected control $=5.0 \pm 0.6$ ) compared with the cag PAI mutants $(12.2 \pm 1.7,3.0 \pm 0.2$-fold; $\mathrm{p}<$ $0.05)$, the oip $A$ mutants $(12.8 \pm 0.7,3.3 \pm 0.2$-fold; $\mathrm{p}<0.05)$, the cag PAI/oipA double mutants $(9.3 \pm 1.1,2.3 \pm 0.06$-fold; $\mathrm{p}<0.01$ ), or heat-killed bacteria $(5.7 \pm 0.4,1.4 \pm 0.05$-fold; $p$ $<0.001$; basal activity for uninfected control $=4.0 \pm 0.4$ ). When we used the p1160-luc + plasmid, similar results were obtained (e.g., normalized activity by Renilla luciferase vector for wild-type $H$. pylori $=17.8 \pm 3.3$, fold increase of uninfected control $=4.9 \pm 0.5$ ). In subsequent experiments, we used p1160-luc+ as the full-length wild-type plasmid when we examined the effects of promoter deletion analyses (all plasmids were derived from PGV-B plasmid [Toyo]) and used -1168 hu.IL6P-luc + as full-length wild-type plasmid when we examined the effects of site mutation analyses (all plasmids were derived from pGL basic plasmid; Promega).

Promoter Deletions and Site Mutation Analyses Showed that AP-1, CRE, C/EBP, and NF-кB Sites were Involved in H. pylori-induced IL-6 Transactivation

To define the regions of the IL- 6 promoter involved in regulating gene expression after $H$. pylori infection, MKN28 cells were transiently transfected with $0.5 \mu \mathrm{g}$ of plasmids containing serial $5^{\prime}$ to $3^{\prime}$ deletions of the IL-6 promoter $(-181,-108$, and -36$)$ linked to the luciferase reporter gene (Figure 6). p181hu.IL6P-luc + plasmid contains binding sites for $C R E, C / E B P$, and NF- $\kappa$ B with deletion of the AP-1 site. p108hu.IL6P-luc + plasmid contains binding sites for NF- $\kappa$ B
Figure 6. IL-6 promoter activation after $H$. pylori infection. MKN28 cells were transiently transfected with $2 \mu \mathrm{g}$ of $5^{\prime}$ deletions of the human IL-6 promoter or with $0.5 \mu \mathrm{g}$ of site mutated human IL-6 promoter, together with $10 \mathrm{ng}$ of Renilla plasmid, and subsequently infected with wild-type $H$. pylori for $9 \mathrm{~h}$. Untreated plates served as controls. For each plate, luciferase activity was normalized to Renilla luciferase vector DNA. Four independent transfections, each run in triplicate, were performed. Data are expressed as fold increase of luciferase activity in H. pylori-infected cells relative to untreated controls. Data are expressed as mean $\pm \mathrm{SE} ;{ }^{*} \mathrm{p}<0.05$, ** $p<0.01$, and *** $p<0.001$ compared with p1160IL6-luc + for deletion analyses and with p1168hu.IL6P-luc + for site mutation analyses.
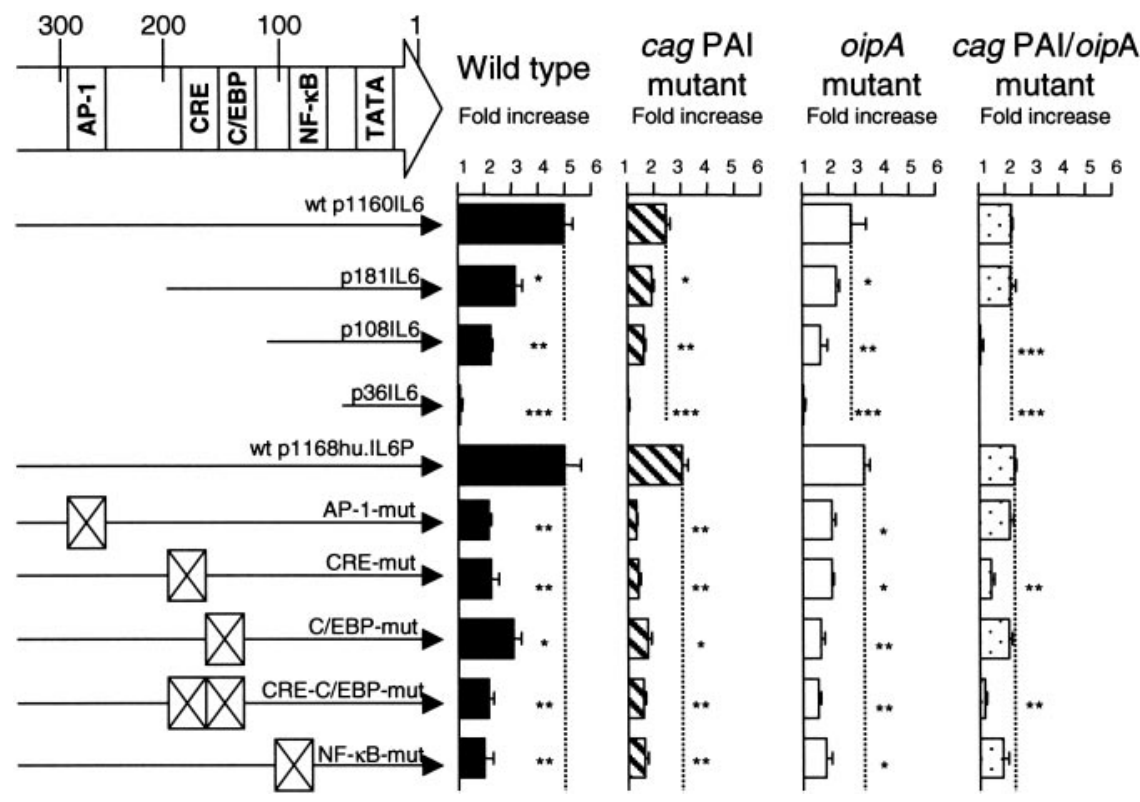
and p36hu.IL6P-luc + plasmid additionally deletes binding site for NF- $\kappa$ B.

Decreased inducibility by wild-type $H$. pylori was observed using the 181-base pair promoter lacking the AP-1 site compared with the full-length p1160-luc + promoter (3.1 \pm 0.3-fold increase of uninfected control; $\mathrm{p}<0.05$; Figure 6). There was no effect on basal activity (normalized activity by Renilla luciferase vector $=3.8 \pm 0.3$ for uninfected control). An additional 5 ' deletion to -108 base pairs, which lacked the CRE and C/EBP sites further reduced H. pyloriinduced luciferase activity $(2.2 \pm 0.08$-fold; $\mathrm{p}<0.01$ from -1168 base pairs) but again with no effect on basal activity (3.6 \pm 0.2$)$. Deletion to -36 base pairs, which additionally lacks the NF- $\kappa \mathrm{B}$ site, abrogated H. pylori-induced luciferase activity $(1.1 \pm 0.09$-fold; $\mathrm{p}<0.001$ from -1168$)$ with marked reduction of the basal activity of the promoter $(0.10 \pm 0.01)$.

As shown in Figure 6, the cag PAI mutants and the oipA mutants produced effects similar to that of the wild-type strains. Deletion to -181 base pairs did not reduce the luciferase activity induced by the cag PAI/oipA double mutants, suggesting that both the cag PAI and OipA play central roles in activating the AP-1 site. A 5' deletion to -108 base pairs reduced the luciferase activity induced by the $c a g$ PAI/oipA double mutants, indicating that factors other than the cag PAI and OipA are involved in activation of the CRE and/or C/EBP sites. Although the luciferase activity induced by the cag PAI/oipA double mutants was relatively weak irrespective of the plasmids used, the results were consistent across five independent experiments. Heat-killed bacteria induced very low luciferase activity (maximum $1.4 \pm 0.05$-fold).

To further characterize the contribution of defined sequence elements, different point-mutated IL-6 promoter variants were tested for their responsively to $H$. pylori. Only site mutations of the AP-1 site or the CRE site reduced the basal activity (unpublished data). Mutation of each binding site significantly reduced the luciferase activity induced by wild-type $H$. pylori, the cag PAI mutants and the oipA mutants (Figure 6). Importantly, mutation of the AP- 1 or NF- $\kappa$ B sites did not affect the activity induced by the cag PAI/oipA double mutants, confirming critical roles for the AP-1 and NF- $\kappa$ B sites in cag PAI- and OipA-induced IL-6 gene transcription. Mutation of the CRE site, but not of the C/EBP site reduced the activity induced by the cag PAI/oipA double mutants, consistent with the notion that factors other than the cag PAI and OipA was involved in activating the CRE site.

\section{Combination of the cag PAI and OipA Play Roles for Activation of NF-кB Site using Plasmids Containing Multimers of IL-6 NF- $\kappa B$}

To further investigate the role(s) of the NF- $\kappa$ B site in H. pylori infection, we used reporter genes containing multimers of the IL-6 NF- $\kappa$ B site ligated upstream of the IL-6 TATA box (Figure 7). Activity of the NF- $\kappa \mathrm{B}$ multimer was highly induced by wild-type strains $(9.7 \pm 1.2$-fold increase of uninfected control; $\mathrm{p}<0.01$ ). The cag PAI mutants and the oipA mutants induced luciferase activity to approximately onethird of that of wild-type strains. The cag PAI/oipA double mutants had little effect on luciferase activity and heat-killed bacteria had no effect on luciferase activity. These data show that the combination of the cag PAI and OipA play important roles in the activation of the NF- $\kappa \mathrm{B}$ site by $H$. pylori infection.

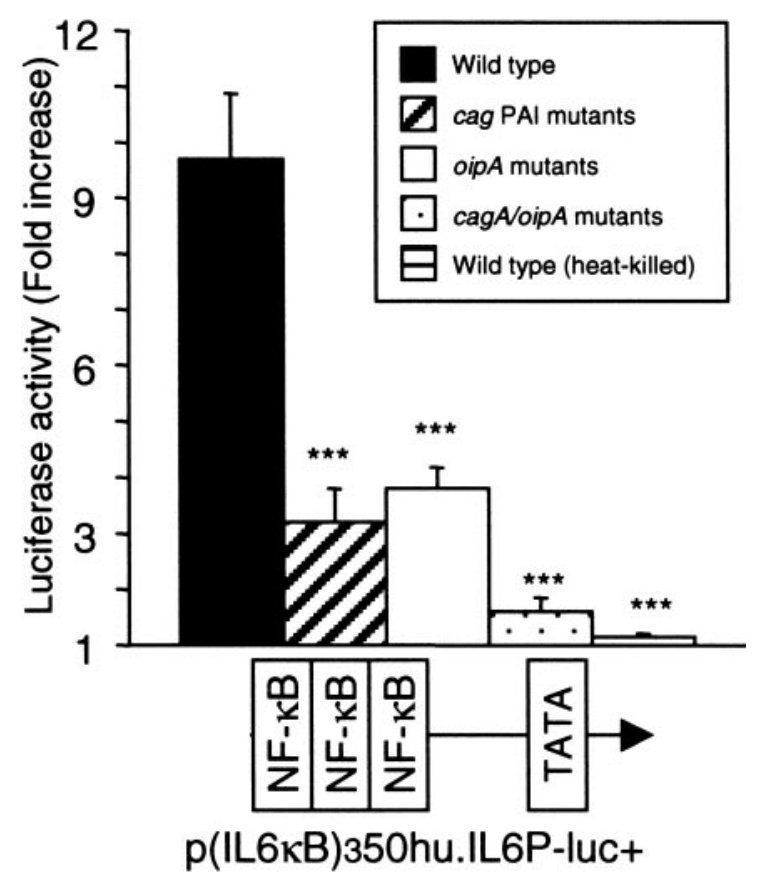

Figure 7. Effect of IL-6 NF- $\kappa$ B multimers after $H$. pylori infection in MKN28 cells. MKN28 cells were cotransfected with $0.5 \mu \mathrm{g}$ of p(IL6kB)350hu.IL6P-luc+ plasmids, together with $10 \mathrm{ng}$ of Renilla plasmid, and subsequently infected with $H$. pylori (wild-type, cag PAI mutants and oipA mutants). Untreated plates served as controls. Shown is the normalized luciferase activity expressed as fold increase of luciferase activity in $H$. pylori-infected cells relative to uninfected controls. Five independent transfections, each run in triplicate, were performed. Data are expressed as mean $\pm \mathrm{SE}$; ${ }_{* * *} \mathrm{p}<0.001$ compared with wild-type H. pylori.

\section{H. pylori-induced IL-6 Promoter Activation Occurred through Cross-talk between MAPK Pathways and IL-6 Specific NF-кB Pathways}

We also used inhibitors for the MAPK pathways and NF- $\kappa$ B pathways to further investigate the effect of the different pathways for luciferase activity. We used full-length plasmid and plasmid containing multimers of the IL-6 NF- $\kappa$ B site (Figure 8, A and B). In agreement with the above protein (Figure 4) and mRNA (Figure 5) results, luciferase assays using full-length plasmid showed that U0126 and SP600125 significantly suppressed the luciferase activity induced by the oipA mutants, but did not affect the activity induced by the cag PAI mutants (Figure 8A). In contrast, SB203580 significantly suppressed luciferase activity induced by the cag PAI mutants but did not suppress the activity induced by the oipA mutants. SB203580 also did not suppress the luciferase activity induced by the cag PAI/oipA double mutants. In contrast, U0126 significantly suppressed the luciferase activity induced by the cag PAI/oipA double mutants. This result confirmed that factors other than the cag PAI and OipA are also involved in activation of the MEK1/2 $\rightarrow$ ERK pathway. MG-132 significantly suppressed luciferase activity of the full-length plasmid using the cag PAI mutants and the oipA mutants, but not when we used the cag PAI/oipA double mutants (Figure 8A). These results are in agreement with the above results showing that the cag PAI and OipA play central roles in $H$. pylori-related NF- $\kappa \mathrm{B}$ activation.

As expected, the activity of the IL- 6 NF- $\kappa$ B multimer induced by H. pylori was abolished by MG-132 (Figure 8B). Surprisingly, SB203580, U0126, and SP600125 also sup- 


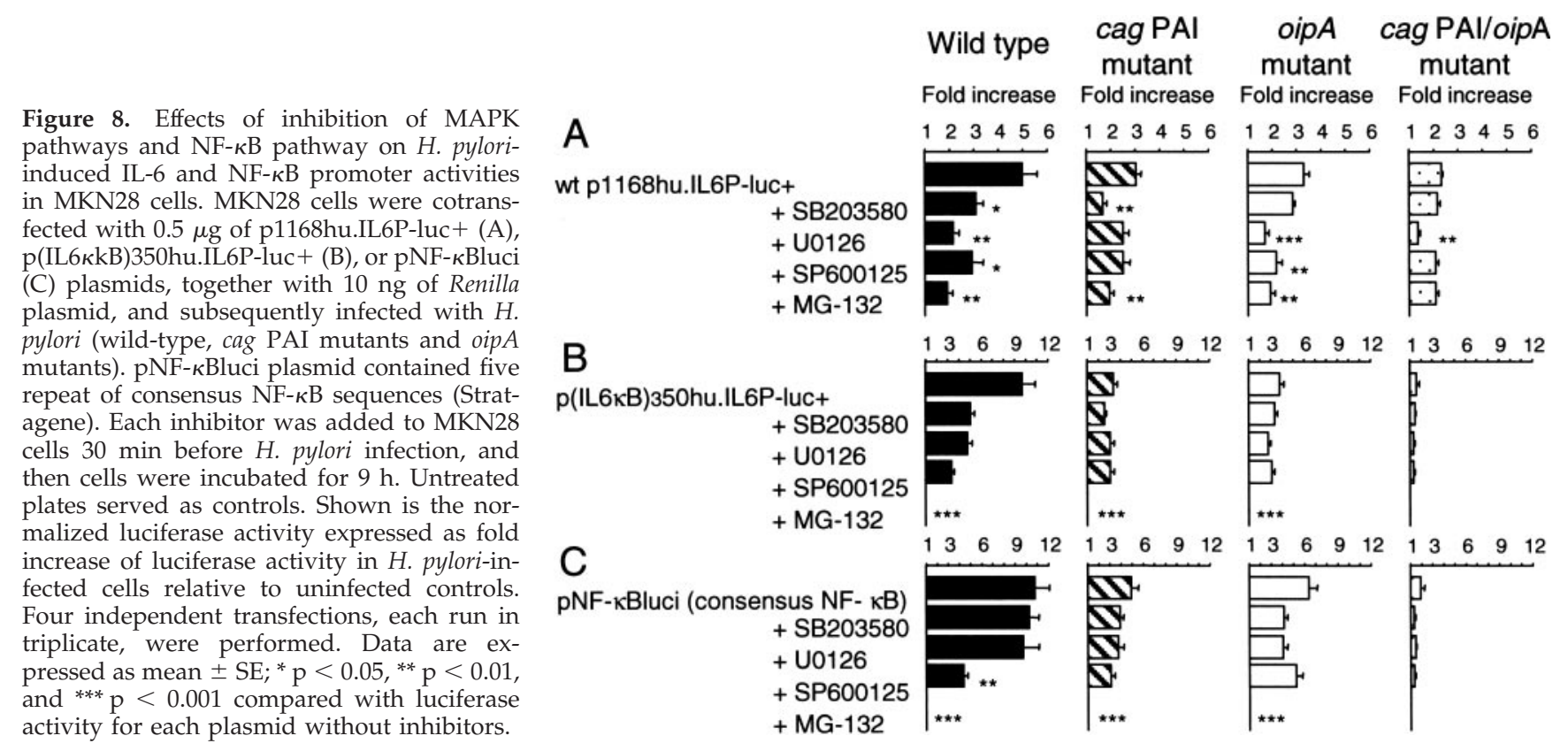

pressed the luciferase activity of the IL- 6 NF- $\kappa$ B multimer induced by wild-type $H$. pylori, suggesting the presence of cross-talk between the MAPK pathways and the IL- 6 NF- $\kappa$ B pathways. To further examine whether the cross-talk was IL-6 NF- $\kappa$ B specific, we used the PathDetect cis-reporting plasmids pNF- $\kappa$ Bluci (Stratagene) that contain the luciferase reporter plasmids with five repeats of the consensus binding sequence for the NF- $\kappa$ B. Importantly, SB203580 and U0126 did not suppress the activity induced by wild-type strains, suggesting that the cross-talk between the p38 and ERK pathways and the NF- $\kappa$ B pathways might be IL-6 specific (Figure 8C). In contrast, SP600125 suppressed the consensus $\mathrm{NF}-\kappa \mathrm{B}$ activity induced by wild-type $H$. pylori, indicating that cross-talk between the JNK pathway and the NF- $\kappa \mathrm{B}$ pathway were general phenomenon in $H$. pylori-infected MKN28 cells.

H. pylori Infection Induced DNA Binding for AP-1, CRE, CIEBP, and NF-кB in IL-6 Promoter and the Binding Was Both the cag PAI and OipA Dependent

Because luciferase reporter gene analysis of the IL-6 promoter showed that binding sites for AP-1, CRE, C/EBP, and $\mathrm{NF}-\kappa \mathrm{B}$ were all involved as regulatory elements in $H$. pyloriinduced $I L-6$ gene transcription, we performed electrophoretic mobility shift assay (EMSA) to determine whether H. pylori infection produced changes in the abundance of DNA-binding proteins recognizing these regions of the promoter. For each binding complex induced by $H$. pylori infection, induction was evident at $1 \mathrm{~h}$ postinfection, peaked at $2 \mathrm{~h}$, and decreased in intensity by $6 \mathrm{~h}$ postinfection (data for $2 \mathrm{~h}$ were shown in Figure 9). The AP-1-binding complex was detected in control MKN28 cells (Figure 9A, lane 1) and further increased after infection with wild-type $H$. pylori (lane 2). The cag PAI mutants, the oipA mutants and the cag $\mathrm{PAI} /$ ip $A$ double mutants reduced binding to AP-1 site compared with wild-type strains (lanes 3-5). Heat-killed bacteria did not induce binding of this site (unpublished data). Binding to the AP-1 site was sequence specific as competition was observed after the addition of unlabeled AP-1 oligonucleotide but not using mutated AP-1 oligonucleotide (lanes 7 and 8). Supershift assays showed that c-Jun bound to the AP-1 site (lanes 10). The anti-c-Fos antibody resulted in a reduction of the binding complexes although clear supershifted bands were not observed, indicating that c-Fos is also a component of the AP-1 complex induced by $H$. pylori infection (lane 11).

As shown in Figure 9B, the CRE-binding complex was not observed in uninfected control cells (lane 1) but was induced after infection with the wild-type $H$. pylori (lane 2). Infection with both the cag PAI mutants and the oipA mutants resulted in reduced binding to this element (lanes 3 and 4). Binding to this site was not abolished by infection with the cag PAI/oipA double mutants (lane 5) confirming that factors other than the cag PAI and OipA are involved in inducing binding to the CRE element. Heat-killed bacteria also induced binding to this site similar to the cag PAI/oipA double mutants (unpublished data). The inducible complex was sequence specific as demonstrated by competition with unlabeled CRE oligonucleotide but not by mutated CRE oligonucleotide (lanes 7 and 8). Supershift assays using anti-c-Fos and anti-c-Jun antibodies resulted in the appearance of a supershifted band and in the disappearance of the CRE complex (lanes 13 and 14). Anti-CREB2 and anti-ATF-2 also resulted in the appearance of a weak supershifted band although there was no apparent reduction of the CRE complex (lanes 11 and 12), suggesting that members of the CREB/ATF family are also components of the H. pyloriinducible CRE complex.

The C/EBP complex was induced after infection with wild-type H. pylori (Figure 9C). Infection with the cag PAI mutants and the oipA mutants resulted in slightly reduced binding to this element (lanes 3 and 4). Similar to the results with the CRE complex, binding to this element was not abolished by infection with the cag PAI/oipA double mutants (lane 5), suggesting that factors other than the cag PAI and OipA are involved in inducing binding to the C/EBP site. Because it was unclear on simple inspection whether reduction occurred, we compared the amount of radioactive probe in the protein-DNA complexes after standardization with free probe. An $\sim 30 \%$ reduction using the isogenic mutants 


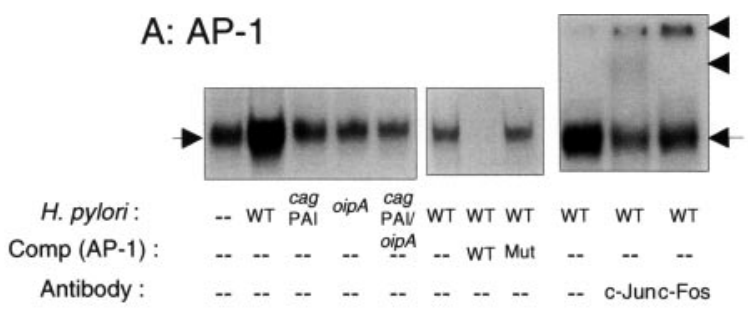

B: CRE

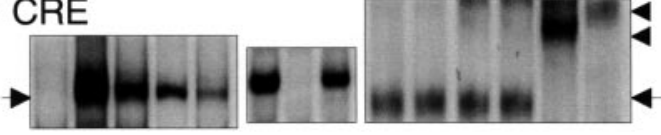

H. pylori: $\quad$-- WT PAl ${ }_{\text {PAPA }}^{\text {cag }}$ PAV WT WT WT WT WT WT WT WT WT

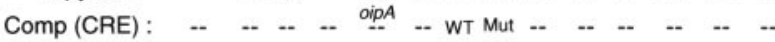

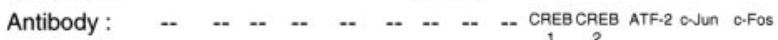

\section{C: C/EBP}

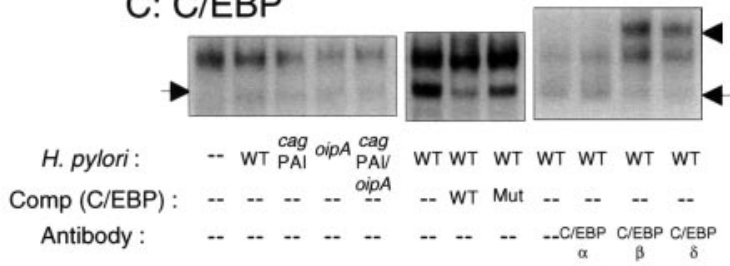

D: NF-кB
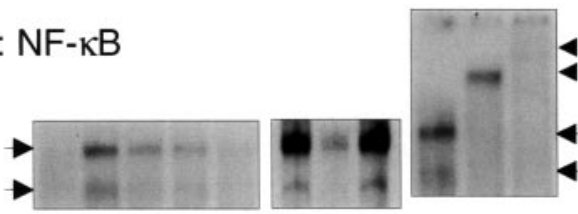

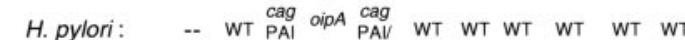

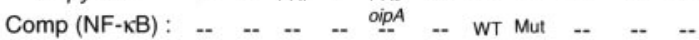

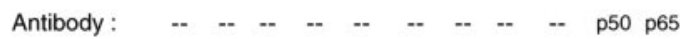

Figure 9. EMSA of IL-6 binding complexes in response to $H$. pylori infection in MKN28 cells. (A) AP-1, (B) CRE, (C) C/EBP, and (D) NF- $\kappa$ B. Lanes $1-5$, nuclear extracts were prepared from control and $H$. pylori-infected MKN28 cells at $2 \mathrm{~h}$ postinfection (wild-type, cag PAI mutants, oipA mutants and cag PAI/oipA double mutants) and used for EMSA. Lanes 6-8, competition analysis. Nuclear extracts from 2-h infected cells were used to bind to the probe in the absence $(-)$ or presence of 100-fold excess of unlabeled competitors (wildtype [WT] or mutated [Mut]) in the binding reaction. Lanes 9 or later, supershift interference assay. Nuclear extracts of MKN45 cells infected for $2 \mathrm{~h}$ were used in the EMSA in the presence of commercial antibodies against specific transcriptional factors.

was shown in four different experiments (unpublished data). Heat-killed bacteria did not induce binding of this site (unpublished data). The inducible complex was sequence specific, as demonstrated by competition with unlabeled C/EBP oligonucleotide but not by mutated C/EBP oligonucleotide (lanes 7 and 8). Supershift assays showed that antiC/EBP- $\beta$ and anti-C/EBP- $\delta$ antibodies resulted in the appearance of a supershifted band (lanes 11 and 12).

As shown in Figure 9D, the NF- $\kappa$ B-binding complex was induced by infection with the wild-type $H$. pylori (lane 2 ). In contrast, the cag PAI mutants and oipA mutants reduced binding to the NF- $\kappa \mathrm{B}$ site compared with wild-type strains (lane 3 and 4). The cag PAI/oipA double mutants induced binding to the NF- $\kappa \mathrm{B}$ site similar to uninfected controls (lane 5). These data confirm the results of the luciferase reporter gene assays showing that both the cag PAI and OipA play central roles in activation of the NF- $\kappa \mathrm{B}$ site. Binding to the
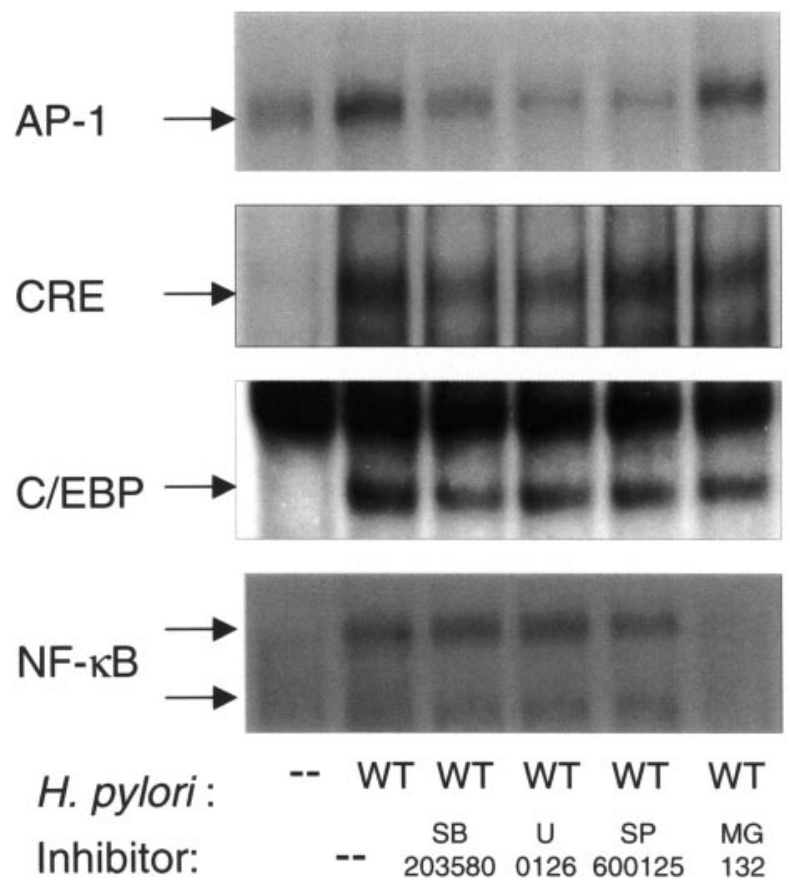

Pathway: $\quad$-- $\quad$ p38 ERK JNK NF-KB

Figure 10. Effects of inhibition of MAPK pathways and NF- $\kappa$ B pathways on $H$. pylori-induced promoter binding in MKN28 cells. Each inhibitor was added to MKN28 cells $30 \mathrm{~min}$ before $H$. pylori infection. Cells were then incubated for $2 \mathrm{~h}$. Nuclear extracts were prepared from control and H. pylori-infected MKN28 cells and used for EMSA. The binding complex of AP-1 was inhibited by each MAPK inhibitor, but not by MG-132. The binding complex of CRE was also inhibited by SB203580 and U0126; however SP600125 and MG-132 had no effect. The binding complex of C/EBP was slightly inhibited by either the MAPK inhibitors or MG-132. The binding complex of NF- $\kappa$ B was inhibited by MG-132; however, it was not inhibited by MAPK inhibitors, suggesting that MAPK inhibitors impeded NF- $\kappa$ B transactivation, but not DNA binding.

NF- $\kappa$ B site was sequence specific as shown by competition with unlabeled NF- $\kappa$ B oligonucleotide but not by mutated NF- $\kappa$ B oligonucleotide (lanes 7 and 8). Supershift assays shows that anti-p50 and anti-p65 antibodies induced the appearance of a super shifted band (lanes 10 and 11).

\section{MAPK Inhibitors Impede NF-кB Transactivation, but not DNA Binding}

We used inhibitors to investigate the pathways used by $H$. pylori to activate the transcription factors of interest. The binding complex of AP-1 was inhibited by all of the MAPK inhibitors tested, but not by MG-132 (Figure 10), confirming that AP-1 binding was regulated by MAPK pathways. The binding complex of CRE was inhibited by SB203580 and U0126; SP600125 and MG-132 had no effect (Figure 10). This result was unexpected considering that c-Jun is a component of the CRE complexes. One possibility is that the JNK inhibitor only impedes transactivation of c-Jun, without influencing binding of c-Jun to the AP-1 site. We compared the amount of radioactive probe in the protein-DNA complexes after standardization with free probe. The binding complex of C/EBP was slightly (30-35\%) inhibited by MAPK inhibitors and MG-132. 


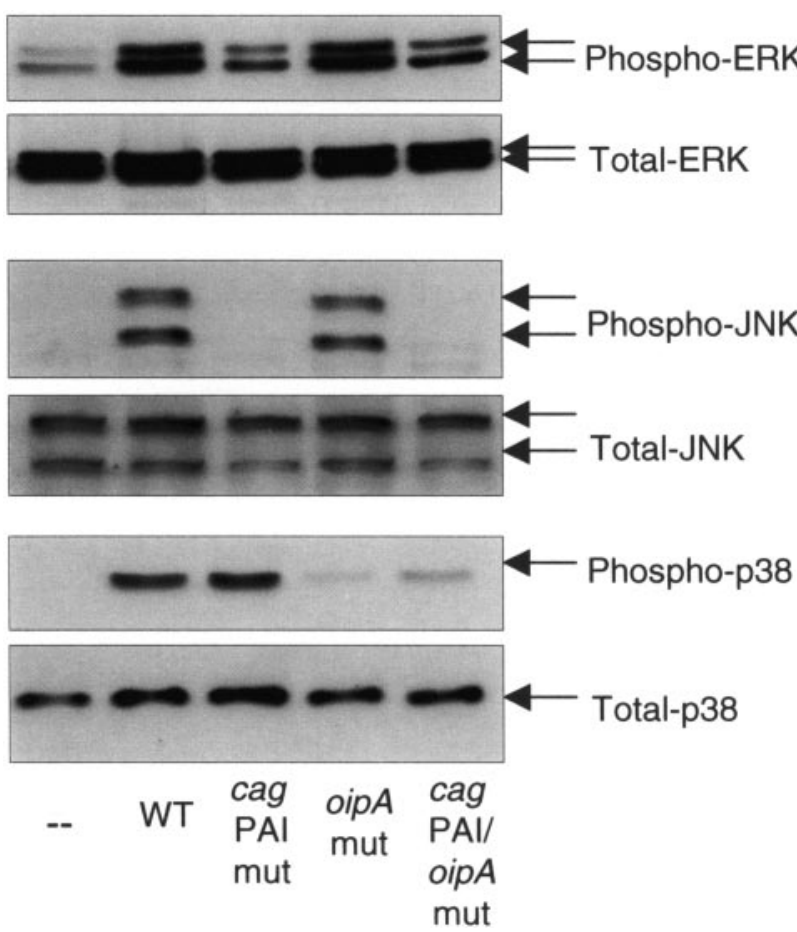

Figure 11. Western blot analysis for MAP kinase antibodies (phospho-specific [upper] and total antibodies). H. pylori (wild-type, cag PAI mutants, oipA mutants and cag PAI/oipA double mutants) were cocultured with MKN28 cells for $120 \mathrm{~min}$. Total protein was extracted and used for Western blot. ERK phosphorylation and JNK phosphorylation were cag PAI dependent and OipA independent. Peak levels of p38 phosphorylation were similar between wild-type and the cag PAI mutants, but were reduced using the oipA mutants.

As expected, the binding complex of NF- $\kappa \mathrm{B}$ was inhibited by MG-132; however it was not inhibited by MAPK inhibitors. This result differs from the results of the luciferase reporter gene assay showing that MAPK inhibitors suppressed the transactivation of NF- $\kappa$ B. This finding suggests that MAPK inhibitors impeded NF- $\kappa$ B transactivation but not DNA binding.

\section{Western Blot Analyses Confirmed that the cag PAI Activated ERK and JNK Pathways, whereas OipA Activated the p38 Pathway}

As inhibitor experiments showed that MAPKs play important roles in IL- 6 gene transcription as well as in IL-6 production, we examined the effect of $H$. pylori infections on phosphorylation of MAPKs using Western blot assays. Wild-type $H$. pylori induced phosphorylation of all three MAPKs in a time-dependent manner with peak levels at $2-4 \mathrm{~h}$ postinfection (the peak levels at $2 \mathrm{~h}$ are shown in Figure 11). That an equal amount of total protein extract in each well was confirmed by Western blot analysis using anti-Actin antibody (sc-1615; Santa Cruz Biotechnology; unpublished data). ERK phosphorylation and JNK phosphorylation were cag PAI dependent and OipA independent (Figure 11). Peak levels of p38 phosphorylation were similar with wild-type and the cag PAI mutants, but were reduced using the oipA mutants. These findings were in agreement with the data for IL-6 mRNA expression (Figure 5), IL-6 protein production (Figure 4) and luciferase reporter gene assays (Figure 8) using the MAPK inhibitors. Overall, the data are consistent with cag PAI $\rightarrow$ ERK/

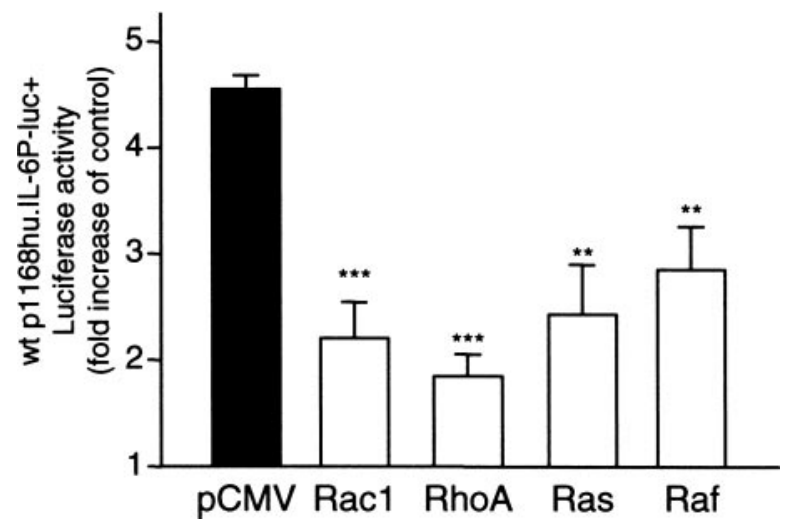

Figure 12. Effects of dominant negative form of monomeric GTPbinding proteins on the induction of the promoter activity of IL- 6 by H. pylori infection. MKN28 cells were cotransfected with $0.5 \mu \mathrm{g}$ of the p1168hu.IL6P-luc + plasmid together with $0.3 \mu \mathrm{g}$ of one of the four plasmids, pCMV-RhoN19, pCMV-RacN17, pCMV-RasN17, or pCMV plus $10 \mathrm{ng}$ of Renilla plasmid as an internal control using LipofectAMINE. The transfected cells were subsequently infected with H. pylori. MKN28 cells were also cotransfected with the p1168hu.IL6P-luc + plasmid together with either $50 \mathrm{ng}$ of pCMV or pCMV-RafS621A plus $10 \mathrm{ng}$ of Renilla plasmid and subsequently infected with $H$. pylori. Untreated plates served as controls. Shown is the normalized luciferase activity expressed as fold increase of luciferase activity in $H$. pylori-infected cells relative to uninfected controls. Five independent transfections, each run in triplicate, were performed. Data are expressed as mean \pm SE; ${ }^{* *} p<0.01$ and ${ }_{* * *} p<0.001$ compared with luciferase activity for the empty vector pCMV. Cotransfection of the dominant negative form of RasN17, RacN19, RhoN19, and RafS621A proteins partially suppressed the induction of the IL-6 promoter by H. pylori infection.

JNK pathways and OipA $\rightarrow$ p38 pathway being involved in IL-6 gene transcription.

\section{GTP-binding Proteins Are Involved in H. pylori-induced IL-6 Transcription}

The upstream factors in the MAPK pathways include monomeric GTP-binding proteins such as Ras, Rac1, and RhoA. To examine the potential role of these $G$ proteins in $H$. pylori-induced IL-6 induction, we tested the capacity of dominant negative proteins RasN17, Rac1N17, and RhoAN19 to inhibit the induction of the IL-6 promoter during $H$. pylori infection. These dominant negative mutants are thought to act by competitively inhibiting the interaction of endogenous GTP-binding proteins with their respective guanine nucleotide exchange factors. MKN28 cells were cotransfected with the p1168hu.IL6P-luc + plasmid $(0.5 \mu \mathrm{g})$ plus one of the following plasmids: pCMV-RasN17, pCMVRacN17, pCMV-RhoN19, or the empty vector pCMV $(0.3 \mu \mathrm{g}$ for each). The transfected cells were subsequently inoculated with $H$. pylori. As shown in Figure 12, cotransfection of the dominant negative form of RasN17, RacN19 and RhoN19 partially suppressed the induction of the IL-6 promoter by $H$. pylori infection. Next, we cotransfected MKN28 cells with the p1168hu.IL6P-luc + plasmid $(0.5 \mu \mathrm{g})$ together with the pCMV-RafS621A (1 $\mu \mathrm{g})$ or with the empty vector pCMV (1 $\mu \mathrm{g})$ and subsequently and then inoculated the transfected cells with $H$. pylori. H. pylori-mediated induction of the promoter activity of IL- 6 was also partially suppressed by the dominant negative protein RafS621A (Figure 12). 


\section{DISCUSSION}

Although many studies have shown that $H$. pylori infection is associated with increased IL-6 production within the gastric mucosa (Crabtree et al., 1991; Yamaoka et al., 1996, 1997, 2001; Ando et al., 1998; Furukawa et al., 1998), the mechanisms involved are largely unresolved. We used an ex vivo cell system of primary gastric epithelial cells to identify the pattern of IL-6 response of the normal human gastric mucosa epithelium to $H$. pylori infection and to the proinflammatory virulence factors, the cag PAI and OipA. The characteristics of the response of normal gastric epithelial cells included: 1) $H$. pylori-induced IL-6 induction was enhanced by both the cag PAI and OipA, 2) both the cag PAI and OipA had similar effects on IL-6 induction, and 3) infection with strains lacking both the cag PAI and OipA did not completely abrogate IL-6 induction. MKN28 cells shared these characteristics and were used for in vitro experiments. Importantly, the nongastric cell line, HeLa cells only met the criteria of cag PAI and OipA dependency, suggesting that generalizability of studies of function of $H$. pylori virulence factors done in nongastric cells and in some gastric cell lines should be viewed with caution.

We explored the signaling pathways upstream of the promoter binding sites and found that the cag PAI and OipA induced IL-6 via different pathways. Our data using MNK28 cells suggests that OipA is involved in the p38 pathways and that the cag PAI is involved in the MEK1/2 $\rightarrow$ ERK and JNK pathways. Use of the dominant negative form of monomeric GTP-binding proteins (Ras, Raf, Rac1, and RhoA) resulted in suppression of the luciferase activity for the IL-6 promoter (Figure 12). Ras is known to be involved in the sequential activation of Raf $\rightarrow$ MEK1/2 $\rightarrow$ ERK (Gille et al., 1992; Brunner et al., 1994; O'Neill et al., 1994). In our present study, AP-1 and C/EBP bindings were inhibited by any of the three MAPK inhibitors. In contrast CRE binding was inhibited by only p38 and MEK1/2 inhibitors. Taken together, these data suggest that the cag PAI pathways involved in IL-6 promoter transcription include Ras $\rightarrow \mathrm{Raf} \rightarrow \mathrm{MEK} 1 / 2 \rightarrow$ ERK $\rightarrow \mathrm{AP}-1 /$ $\mathrm{CRE} / \mathrm{C} / \mathrm{EBP}$ and JNK $\rightarrow \mathrm{AP}-1 / \mathrm{C} / \mathrm{EBP}$ pathways and OipA pathways include $\mathrm{p} 38 \rightarrow \mathrm{AP}-1 / \mathrm{CRE} / \mathrm{C} / \mathrm{EBP}$.

Recent studies have shown that RhoA/Rac-dependent activation of NF- $\kappa \mathrm{B}$ in gastric cells induced by H. pylori (Wroblewski et al., 2003; Varro et al., 2004). We showed that both the cag PAI and OipA were involved in NF- $\kappa$ B pathway and we speculate that, both the cag PAI and OipA may be involved in RhoA/Rac $1 \rightarrow \mathrm{NF}-\kappa \mathrm{B}$ pathways.

Interestingly, each of the three MAPK inhibitors suppressed the luciferase activity of the $H$. pylori-induced IL-6 NF- $\kappa \mathrm{B}$ multimer, suggesting the possibility of cross-talk between the MAPK pathways and NF- $\kappa$ B pathways. It appears that cross-talk between the p38 or ERK pathways and NF- $\kappa$ B pathway was IL-6 NF- $\kappa$ B specific as p38 and ERK inhibitors did not suppress the $H$. pylori-induced luciferase activity of the consensus NF- $\kappa \mathrm{B}$ multimer (Figure 8). To our knowledge, this is the first study to show cross-talk between MAPK pathways and the NF- $\kappa$ B pathway in $H$. pylori-infected gastric epithelial cells. Importantly, none of the three MAPK inhibitors affected NF- $\kappa$ B DNA-binding activity, suggesting that the MAPKs do not affect $H$. pylori-induced NF- $\kappa$ B DNA-binding activity, but do induce NF- $\kappa$ B transactivation. This is a novel finding in $H$. pylori-infected gastric epithelial cells and is in agreement with a recent study in mouse fibroblasts where MAPK inhibitors had no effect on TNF-induced NF- $\kappa$ B DNA-binding activity although each inhibitor (p38 and ERK inhibitors) suppressed the transac- tivation of NF- $\kappa \mathrm{B}$ (Beyaert et al., 1996; Vanden Berghe et al., 2000; Vermeulen et al., 2003). Those mouse fibroblast studies resulted in a proposed model, suggesting that NF- $\kappa \mathrm{B}$ acts as final trigger to activate a multiprotein complex, a so-called "enhanceosome" at the level of the IL-6 promoter with MAPK activity beings unequivocally linked to the histone acetylation capacity of the enhanceosome to stimulate gene expression in response to TNF (Vermeulen et al., 2003). Similar mechanisms might also apply to $H$. pylori-induced IL-6 promoter activation. Further studies will be necessary to investigate $H$. pylori-induced activation of the IL- 6 enhanceosome.

Interestingly, the mechanisms of $H$. pylori-induced IL-6 induction differed from those involved in the induction of the proinflammatory cytokine, IL-8 (Aihara et al., 1997; Keates et al., 1999; Naumann et al., 1999; Meyer-ter-Vehn et al., 2000; Wessler et al., 2000, 2002; Keates et al., 2001; Yamaoka et al., 2004). For example, H. pylori-induced full activation of the IL-6 promoter involved binding sites for AP-1, CRE, C /EBP, and NF- $\kappa$ B, whereas $H$. pylori-associated IL-8 promoter activation involved the AP-1 and NF- $\kappa$ B sites but not the C/EBP (NF-IL6) sites (Aihara et al., 1997; Yamaoka et al., 2004). We showed the cag PAI and OipA were separately involved in activation of all four binding sites (Figures 6 and 9). These results also differ from activation of the IL-8 promoter where the cag PAI, but not OipA, is involved in activation of the AP-1 and NF- $\kappa$ B sites (Yamaoka et al., 2004). Both the cag PAI mutants and the oipA mutants produced similar effects with regard to the IL-6 protein and mRNA, leading us to conclude that they have similar effects on IL-6 induction. These results contrast with studies on IL-8 induction where the cag PAI had a much more prominent effect than did OipA (Akanuma et al., 2002; Ando et al., 2002; Odenbreit et al., 2002; Yamaoka et al., 2002, 2004). These differences are consistent with the data showing OipA-induced activation of both the AP- 1 and NF- $\kappa$ B sites of the IL- 6 promoter but has no effect on either site in the IL- 8 promoter. A final difference was that the cag PAI/oipA double mutants or heat-killed bacteria were able to induce small amounts of IL-6, but not IL-8, from gastric epithelial cells (Aihara et al., 1997; Keates et al., 1999, 2001; Yamaoka et al., 2004). Inhibitor experiments showed that the MEK1/2 $\rightarrow$ ERK pathways were involved in IL-6 induction by heat-killed bacteria (Figures 4 and 5). EMSA showed that heat-killed bacteria were involved in the binding to the CRE site. Taken together, the data suggest that the heat-resistant factors that are unrelated to the cag PAI and OipA induced IL-6 via MEK1/2 $\rightarrow$ ERK $\rightarrow$ CRE pathways.

Our results showing that $H$. pylori-associated IL-6 induction differs from IL- 8 induction is also consistent with findings in vivo. For example, IL-8 mRNA/protein levels in gastric biopsy specimens are closely related to the $\operatorname{cag} A$ status, whereas the relationship between IL-6 mRNA/protein levels and the $\operatorname{cag} A$ status is weak (Yamaoka et al., 1996, 1997). Another difference is that in vivo the mucosal IL-8 levels rapidly normalize after $H$. pylori eradication, whereas IL-6 levels decrease gradually (Ando et al., 1998). In addition, IL-6 mRNA levels, but not IL-8 mRNA levels were overexpressed at the either the margin of $H$. pylori-related gastric ulcers (Furukawa et al., 1998) and in early stage gastric cancer (Yamaoka et al., 2001). The different mechanisms of IL-6 induction from IL-8 induction should give us a new insight for $H$. pylori-associated gastroduodenal pathogenesis. 


\section{ACKNOWLEDGMENTS}

We thank Dr. Antonia R. Sepulveda (Department of Pathology, University of Pittsburgh) for providing SNU638 and SNU668 cells, Dr. Masafumi Nakao (Takeda Chemical Industries, Osaka, Japan) for providing H. pylori strain TN2GF4, and Dr. Toshio Hirano (Osaka University Medical School, Osaka, Japan) for providing various IL-6 promoter plasmids. We also thank Dr. Rainer Haas for providing the kanamycin resistance gene cassette and Dr. Diane E. Taylor for providing the chloramphenicol resistance gene cassette. This material is based on work supported in part by National Institutes of Health Grants R01 DK62813 (Y.Y.), by the Office of Research and Development Medical Research Service Department of Veterans Affairs (D.Y.G.), and by Public Health Service Grant DK56338, which funds the Texas Gulf Coast Digestive Diseases Center.

\section{REFERENCES}

Aihara, M., Tsuchimoto, D., Takizawa, H., Azuma, A., Wakebe, H., Ohmoto, Y., Imagawa, K., Kikuchi, M., Mukaida, N., and Matsushima, K. (1997). Mechanisms involved in Helicobacter pylori-induced interleukin-8 production by a gastric cancer cell line, MKN45. Infect. Immun. 65, 3218-3224.

Akanuma, M. et al. (2002). The evaluation of putative virulence factors of Helicobacter pylori for gastroduodenal disease by use of a short-term Mongolian gerbil infection model. J. Infect. Dis. 185, 341-347.

Akira, S. (1997). IL-6-regulated transcription factors. Int. J. Biochem. Cell Biol. 29, 1401-1418.

Ando, T. et al. (1998). Differential normalization of mucosal interleukin-8 and interleukin-6 activity after Helicobacter pylori eradication. Infect. Immun. 66, 4742-4747.

Ando, T., Peek, R. M., Jr., Lee, Y. C., Krishna, U., Kusugami, K., and Blaser, M. J. (2002). Host cell responses to genotypically similar Helicobacter pylor isolates from United States and Japan. Clin. Diagn. Lab. Immunol. 9, 167-175.

Atherton, J. C., Cao, P., Peek, R. M., Jr., Tummuru, M. K., Blaser, M. J., and Cover, T. L. (1995). Mosaicism in vacuolating cytotoxin alleles of Helicobacter pylori. Association of specific vacA types with cytotoxin production and peptic ulceration. J. Biol. Chem. 270, 17771-17777.

Beyaert, R., Cuenda, A., Vanden Berghe, W., Plaisance, S., Lee, J. C., Haegeman, G., Cohen, P., and Fiers, W. (1996). The p38/RK mitogen-activated protein kinase pathway regulates interleukin- 6 synthesis response to tumor necrosis factor. EMBO J. 15, 1914-1923.

Brasier, A. R., Jamaluddin, M., Casola, A., Duan, W., Shen, Q., and Garofalo, R. P. (1998). A promoter recruitment mechanism for tumor necrosis factoralpha-induced interleukin-8 transcription in type II pulmonary epithelial cells. Dependence on nuclear abundance of Rel A, NF-kappaB1, and c-Rel transcription factors. J. Biol. Chem. 273, 3551-3561.

Brunner, D., Ducker, K., Oellers, N., Hafen, E., Scholz, H., and Klambt, C. (1994). The ETS domain protein pointed-P2 is a target of MAP kinase in the sevenless signal transduction pathway. Nature 370, 386-389.

Censini, S., Lange, C., Xiang, Z., Crabtree, J. E., Ghiara, P., Borodovsky, M., Rappuoli, R., and Covacci, A. (1996). cag, a pathogenicity island of Helicobacter pylori, encodes type I-specific and disease-associated virulence factors. Proc. Natl. Acad. Sci. USA 93, 14648-14653.

Coso, O. A., Chiariello, M., Yu, J. C., Teramoto, H., Crespo, P., Xu, N., Miki, T., and Gutkind, J. S. (1995). The small GTP-binding proteins Rac1 and Cdc42 regulate the activity of the JNK/SAPK signaling pathway. Cell 81, 1137-1146.

Crabtree, J. E., Shallcross, T. M., Heatley, R. V., and Wyatt, J. I. (1991). Mucosal tumour necrosis factor alpha and interleukin-6 in patients with Helicobacter pylori associated gastritis. Gut 32, 1473-1477.

Crawford, H. C., Krishna, U. S., Israel, D. A., Matrisian, L. M., Washington, M. K., and Peek, R. M., Jr. (2003). Helicobacter pylori strain-selective induction of matrix metalloproteinase-7 in vitro and within gastric mucosa. Gastroenterology $125,1125-1136$.

Frost, R. A., Nystrom, G. J., and Lang, C. H. (2003). Lipopolysaccharide and proinflammatory cytokines stimulate interleukin-6 expression in $\mathrm{C} 2 \mathrm{C} 12 \mathrm{myo}-$ blasts: role of the Jun NH2-terminal kinase. Am. J. Physiol. Regul. Integr. Comp. Physiol. 285, R1153-R1164.

Furukawa, K., Takahashi, T., Arai, F., Matsushima, K., and Asakura, H. (1998). Enhanced mucosal expression of interleukin- 6 mRNA but not of interleukin- 8 mRNA at the margin of gastric ulcer in Helicobacter pylori-positive gastritis. J. Gastroenterol. 33, 625-633.

Gille, H., Sharrocks, A. D., and Shaw, P. E. (1992). Phosphorylation of transcription factor p62TCF by MAP kinase stimulates ternary complex formation at c-fos promoter. Nature $358,414-417$.
Gobert, A. P., Bambou, J. C., Werts, C., Balloy, V., Chignard, M., Moran, A. P., and Ferrero, R. L. (2004). Helicobacter pylori heat shock protein 60 mediates interleukin-6 production by macrophages via a toll-like receptor (TLR)-2TLR-4-, and myeloid differentiation factor 88-independent mechanism. J. Biol. Chem. 279, 245-250.

Heuermann, D., and Haas, R. (1998). A stable shuttle vector system for efficient genetic complementation of Helicobacter pylori strains by transformation and conjugation. Mol. Gen. Genet. 257, 519-528.

Hwang, I. R., Hsu, P. I., Peterson, L. E., Gutierrez, O., Kim, J. G., Graham, D. Y., and Yamaoka, Y. (2003). Interleukin-6 genetic polymorphisms are not related to Helicobacter pylori-associated gastroduodenal diseases. Helicobacter 8, 142-148.

Keates, S., Keates, A. C., Warny, M., Peek, R. M., Jr., Murray, P. G., and Kelly, C. P. (1999). Differential activation of mitogen-activated protein kinases in AGS gastric epithelial cells by cag + and cag - Helicobacter pylori. J. Immunol. 163, 5552-5559.

Keates, S., Sougioultzis, S., Keates, A. C., Zhao, D., Peek, R. M., Jr., Shaw, L. M., and Kelly, C. P. (2001). cag + Helicobacter pylori induce transactivation of the epidermal growth factor receptor in AGS gastric epithelial cells. J. Biol. Chem. 276, 48127-48134

Kudo, T., Nurgalieva, Z. Z., Conner, M. E., Crawford, S., Odenbreit, S., Haas, R., Graham, D. Y., and Yamaoka, Y. (2004). Correlation between Helicobacter pylori OipA protein expression and oipA gene switch status. J. Clin. Microbiol. 42, 2279-2281.

Meyer-ter-Vehn, T., Covacci, A., Kist, M., and Pahl, H. L. (2000). Helicobacter pylori activates mitogen-activated protein kinase cascades and induces expression of the proto-oncogenes c-fos and c-jun. J. Biol. Chem. 275, 16064-16072.

Muraoka, O., Kaisho, T., Tanabe, M., and Hirano, T. (1993). Transcriptional activation of the interleukin-6 gene by HTLV-1 p40tax through an NF-kappa B-like binding site. Immunol. Lett. 37, 159-165.

Naumann, M., Wessler, S., Bartsch, C., Wieland, B., Covacci, A., Haas, R., and Meyer, T. F. (1999). Activation of activator protein 1 and stress response kinases in epithelial cells colonized by Helicobacter pylori encoding the cag pathogenicity island. J. Biol. Chem. 274, 31655-31662.

Odenbreit, S., Kavermann, H., Puls, J., and Haas, R. (2002). CagA tyrosine phosphorylation and interleukin- 8 induction by Helicobacter pylori are independent from alpAB, HopZ and bab group outer membrane proteins. Int J. Med. Microbiol. 292, 257-266.

O'Neill, E. M., Rebay, I., Tjian, R., and Rubin, G. M. (1994). The activities of two Ets-related transcription factors required for Drosophila eye development are modulated by the Ras/MAPK pathway. Cell 78, 137-147.

Plaisance, S., Vanden Berghe, W., Boone, E., Fiers, W., and Haegeman, G. (1997). Recombination signal sequence binding protein Jkappa is constitutively bound to the NF-kappaB site of the interleukin- 6 promoter and acts as a negative regulatory factor. Mol. Cell Biol. 17, 3733-3743.

Pritts, T. A., Hungness, E. S., Hershko, D. D., Robb, B. W., Sun, X., Luo, G. J., Fischer, J. E., Wong, H. R., and Hasselgren, P. O. (2002). Proteasome inhibitors induce heat shock response and increase IL-6 expression in human intestinal epithelial cells. Am. J. Physiol. Regul. Integr. Comp. Physiol. 282, R1016R1026.

Shibata, T., Imaizumi, T., Tamo, W., Matsumiya, T., Kumagai, M., Cui, X. F., Yoshida, H., Takaya, S., Fukuda, I., and Satoh, K. (2002). Proteasome inhibitor MG-132 enhances the expression of interleukin-6 in human umbilical vein endothelial cells: Involvement of MAP/ERK kinase. Immunol. Cell Biol. 80, $226-230$.

Sun, Y., Wenger, L., Brinckerhoff, C. E., Misra, R. R., and Cheung, H. S. (2002) Basic calcium phosphate crystals induce matrix metalloproteinase-1 through the Ras/mitogen-activated protein kinase/c-Fos/AP-1/metalloproteinase 1 pathway. Involvement of transcription factor binding sites AP-1 and PEA-3. J. Biol. Chem. 277, 1544-1552.

Tanahashi, T., Kita, M., Kodama, T., Yamaoka, Y., Sawai, N., Ohno, T., Mitsufuji, S., Wei, Y. P., Kashima, K., and Imanishi, J. (2000). Cytokine expression and production by purified Helicobacter pylori urease in human gastric epithelial cells. Infect. Immun. 68, 664-671.

Vanden Berghe, W., De Bosscher, K., Boone, E., Plaisance, S., and Haegeman, G. (1999). The nuclear factor-kappaB engages CBP/p300 and histone acetyltransferase activity for transcriptional activation of the interleukin- 6 gene promoter. J. Biol. Chem. 274, 32091-32098.

Vanden Berghe, W., Plaisance, S., Boone, E., De Bosscher, K., Schmitz, M. L., Fiers, W., and Haegeman, G. (1998). p38 and extracellular signal-regulated kinase mitogen-activated protein kinase pathways are required for nuclear factor-kappaB p65 transactivation mediated by tumor necrosis factor. J. Biol. Chem. 273, 3285-3290. 
Vanden Berghe, W., Vermeulen, L., De Wilde, G., De Bosscher, K., Boone, E., and Haegeman, G. (2000). Signal transduction by tumor necrosis factor and gene regulation of the inflammatory cytokine interleukin-6. Biochem. Pharmacol. 60, 1185-1195.

Varro, A., Noble, P. J., Pritchard, D. M., Kennedy, S., Hart, C. A., Dimaline, R., and Dockray, G. J. (2004). Helicobacter pylori induces plasminogen activator inhibitor 2 in gastric epithelial cells through nuclear factor-kappaB and RhoA: implications for invasion and apoptosis. Cancer Res. 64, 1695-1702.

Vermeulen, L., De Wilde, G., Van Damme, P., Vanden Berghe, W., and Haegeman, G. (2003). Transcriptional activation of the NF-kappaB p65 subunit by mitogen- and stress-activated protein kinase-1 (MSK1). EMBO J. 22, 1313-1324.

Watanabe, T., Tada, M., Nagai, H., Sasaki, S., and Nakao, M. (1998). Helicobacter pylori infection induces gastric cancer in mongolian gerbils. Gastroenterology $115,642-648$

Wessler, S., Hocker, M., Fischer, W., Wang, T. C., Rosewicz, S., Haas, R., Wiedenmann, B., Meyer, T. F., and Naumann, M. (2000). Helicobacter pylor activates the histidine decarboxylase promoter through a mitogen-activated protein kinase pathway independent of pathogenicity island-encoded virulence factors. J. Biol. Chem. 275, 3629-3636.

Wessler, S., Rapp, U. R., Wiedenmann, B., Meyer, T. F., Schoneberg, T., Hocker, M., and Naumann, M. (2002). B-Raf/Rap1 signaling, but not c-Raf$1 /$ Ras, induces the histidine decarboxylase promoter in Helicobacter pylori infection. FASEB J. 16, 417-419.
Wroblewski, L. E., Noble, P. J., Pagliocca, A., Pritchard, D. M., Hart, C. A., Campbell, F., Dodson, A. R., Dockray, G. J., and Varro, A. (2003). Stimulation of MMP-7 (matrilysin) by Helicobacter pylori in human gastric epithelial cells: role in epithelial cell migration. J. Cell Sci. 116, 3017-3026.

Yamaoka, Y., Kikuchi, S., el-Zimaity, H. M., Gutierrez, O., Osato, M. S., and Graham, D.Y. (2002). Importance of Helicobacter pylori oipA in clinical presentation, gastric inflammation, and mucosal interleukin 8 production. Gastroenterology 123, 414-424.

Yamaoka, Y., Kita, M., Kodama, T., Sawai, N., and Imanishi, J. (1996). Helicobacter pylori cagA gene and expression of cytokine messenger RNA in gastric mucosa. Gastroenterology 110, 1744-1752.

Yamaoka, Y., Kita, M., Kodama, T., Sawai, N., Kashima, K., and Imanishi, J. (1997). Induction of various cytokines and development of severe mucosa inflammation by $\operatorname{cag} A$ gene positive Helicobacter pylori strains. Gut 41, 442 451

Yamaoka, Y., Kodama, T., Kita, M., Imanishi, J., Kashima, K., and Graham, D. Y. (2001). Relation between cytokines and Helicobacter pylori in gastric cancer. Helicobacter 6, 116-124.

Yamaoka, Y., Kudo, T., Lu, H., Casola, A., Brasier, A. R., and Graham, D. Y. (2004). Role of interferon-stimulated responsive element-like element in interleukin-8 promoter in Helicobacter pylori infection. Gastroenterology 126, 1030-1043.

Yamaoka, Y., Kwon, D. H., and Graham, D. Y. (2000). A M(r) 34,000 proinflammatory outer membrane protein (oipA) of Helicobacter pylori. Proc. Natl. Acad. Sci. USA 97, 7533-7538. 